\title{
Quantum mechanics on a circle: Husimi phase-space distributions and semiclassical coherent state propagators
}

\author{
B Bahr ${ }^{1}$ and H J Korsch ${ }^{2}$ \\ ${ }^{1}$ Max-Planck Institute for Gravitational Physics (Albert-Einstein Institute), Am Mühlenberg 1, \\ D-14476 Golm, Germany \\ ${ }^{2}$ Technical University of Kaiserslautern, Gottlieb-Daimler-Straße, D-67663 Kaiserslautern, \\ Germany \\ E-mail: bbahr@aei.mpg.de
}

Received 10 November 2006, in final form 13 February 2007

Published 20 March 2007

Online at stacks.iop.org/JPhysA/40/3959

\begin{abstract}
We discuss some basic tools for an analysis of one-dimensional quantum systems defined on a cyclic coordinate space. The basic features of the generalized coherent states, the complexifier coherent states, are reviewed. These states are then used to define the corresponding (quasi)densities in phase space. The properties of these generalized Husimi distributions are discussed, in particular their zeros. Furthermore, the use of the complexifier coherent states for a semiclassical analysis is demonstrated by deriving a semiclassical coherent state propagator in phase space.
\end{abstract}

PACS numbers: $03.65 .-\mathrm{w}, 03.65 . \mathrm{Sq}$

\section{Introduction}

Quantum systems depending on a cyclic coordinate appear in numerous studies. Celebrated examples of such systems are the particle on a circle, the plane pendulum, the rotor, but also other systems such as space periodic crystals in a tight-binding approximation, where the cyclic variable is the quasimomentum [1]. Despite much effort, the theory of quantization of such systems is still far from complete (see, e.g., [2] and references therein). In the present paper, we do not aim at a discussion of the still interesting issue of angle operators and related problems. We will concentrate on an important tool for a theoretical analysis of cyclic quantum systems, the generalized coherent states.

The coherent states on the real line provide a quite useful tool for a semiclassical analysis of one-dimensional systems. Their generalization to field theory is the main tool for describing 
laser light and other states of coherently superposed matter. This has raised the question if one can find states with equally pleasant properties for other systems, such as quantum mechanic on the circle [2-5], or for other dynamical groups, such as the states defined by Perelomov or Gilmore [6].

In [7], a method was described to construct coherent states for quantum mechanics on any compact, connected Lie group $G$. The mathematical foundation for this was laid in [810]. The procedure relies on the fact that the tangent bundle $T^{*} G$, which can be identified with the phase space of a particle moving on $G$, is diffeomorphic to the complexification $G^{\mathbb{C}}$ of $G$, which named these states 'complexifier coherent states'. Their general properties have been exhibited in [11-13]. For $G=S U(2)$, the complexifier coherent states are used for the semiclassical analysis of loop quantum gravity [11], and the states for $G=U(1)$ and $G=U(1)^{3}$ are employed in the semiclassical analysis of quantum cosmology [14] and linearized quantum gravity [7].

The complexifier procedure could also be used to define coherent states on $d$-dimensional spheres $S^{d}[18]$.

For the special case of $G=U(1)$, i.e. quantum mechanics on the circle, the complexifier coherent states reduce to the already employed coherent states on the circle, which had either been derived from a generalization of the harmonic oscillator coherent states $[3,5,16]$, or simply guessed $[15,20]$. In [2], one can find an extensive description of these states and their properties.

In this paper, we will shortly review the most important properties of the complexifier coherent states for $G=U(1)$ and demonstrate that these states can be used for a semiclassical analysis of quantum mechanics on the circle, towards which we will pursue two different ways.

First, we will analyse the Bargmann-Segal representation of states on the circle, defined by the complexifier coherent states. In particular, this enables one to study the dynamics of one-dimensional periodic systems by the zeros of the Husimi distribution. For quantum mechanical systems on the real line the Husimi distribution is a well-developed tool to study, e.g., quantum chaos $[17,20]$. The Husimi distribution for systems with cyclic coordinate is a special case of a class of phase-space (quasi)densities that have been recently investigated [21]. It can be defined with the help of the complexifier coherent states in complete analogy to the Husimi distributions for systems on the real line.

In our work, we will show that one needs even less effort to reconstruct the state from its zeros than in the case of quantum mechanics on the real line. This could serve as a starting point of investigating quantum chaotic behaviour of periodic systems, such as happened for the driven rotor [19].

In the second part of this paper, we derive the semiclassical propagator on a cylindrical phase space, which is the transition amplitude $\left\langle z_{F}|\exp (-\mathrm{i} \hat{H} \tau)| z_{I}\right\rangle$ between complexifier coherent states. This has already been done for the special case of the freely moving particle $[2,23,24]$, but we will do the analysis for arbitrary Hamiltonians.

Semiclassical propagators have a long history and have been studied in detail, e.g. for systems on the real line, without or with spin (see, e.g., [22, 25-29] and references therein). They are a main ingredient for the semiclassical analysis of quantum systems and a powerful tool to investigate the transition from quantum-to-classical behaviour of systems. The derivation of the semiclassical propagator on the circle follows similar lines as the one for the propagator on the real line [22]. Finally, the propagator on the circle can be represented as an infinite sum over propagators on the real line, representing different winding numbers of paths in $U(1)$, which nicely demonstrates the influence of the global topological properties of phase space on the quantum dynamics. 


\section{Quantum mechanics on the circle}

The phase space for a classical particle moving on a circle $U(1)$ is given by $T^{*}\left(S^{1}\right) \simeq S^{1} \times \mathbb{R}$. Since the angle $\phi$ is not periodic, it is no phase-space function. So one has to work with $\exp (\mathrm{i} \phi)$, of which one can compute $\phi$ only modulo $2 \pi$. Together with the canonical momentum $p$, which has the dimension of angular momentum, the Poisson bracket

$$
\{f, g\}=\frac{\partial f}{\partial \phi} \frac{\partial g}{\partial p}-\frac{\partial g}{\partial \phi} \frac{\partial f}{\partial p}
$$

implies

$$
\{\exp (\mathrm{i} \phi), p\}=\mathrm{i} \exp (\mathrm{i} \phi) .
$$

Note that, although a function on phase space, $\exp (\mathrm{i} \phi)$ is not an observable, since it is not real valued.

Quantization is achieved by replacing observables by self-adjoint operators and Poisson brackets by commutators divided by $\mathrm{i} \hbar$. Since $\exp (\mathrm{i} \phi)$ is not a real-valued function, but takes values in $U(1)$, we will replace it by a unitary operator $\exp (\mathrm{i} \hat{\phi})$. The quantization of (2.2) then yields

$$
[\exp (\mathrm{i} \hat{\phi}), \hat{p}]=\mathrm{i} \hbar(\mathrm{i} \exp (\mathrm{i} \hat{\phi}))=-\hbar \exp (\mathrm{i} \hat{\phi}) .
$$

The quantization of (2.2) is nontrivial, since there are infinitely many unitarily inequivalent representations of $(2.3)$ [2,3]. The different representations live all on the Hilbert space

$$
\mathcal{H}=L^{2}[0,2 \pi],
$$

with the inner product

$$
\langle\psi, \varphi\rangle=\int_{0}^{2 \pi} \frac{\mathrm{d} \phi}{2 \pi} \overline{\psi(\phi)} \varphi(\phi),
$$

where the operator $\exp (\mathrm{i} \hat{\phi})$ acts as multiplication

$$
(\exp (\mathrm{i} \hat{\phi}) \psi)(\phi):=\exp (\mathrm{i} \phi) \psi(\phi),
$$

and $\hat{p}$ as differentiation

$$
(\hat{p} \psi)(\phi)=\frac{\hbar}{\mathrm{i}} \frac{\mathrm{d} \psi}{\mathrm{d} \phi}(\phi) .
$$

While $\exp (\mathrm{i} \hat{\phi})$ is a unitary operator, hence bounded by one, it is defined everywhere, whereas $\hat{p}$ is unbounded, and one has to worry about domains of definition. In particular, the inequivalent representations of (2.3) differ by the dense domain of the definition of $\hat{p}$. They are labelled by a real parameter $0 \leqslant \delta<1$. Fixing $\delta$, one defines a basis of $\mathcal{H}$ by

$$
|n\rangle_{\delta}=\phi \mapsto \exp (\mathrm{i}(n+\delta) \phi), \quad n \in \mathbb{Z} .
$$

The domain of definition for $\hat{p}$ in the representation labelled by $\delta$ is the one given by the following set of linear combinations of $|n\rangle_{\delta}$ :

$$
\mathcal{D}_{\hat{p}}^{\delta}:=\left\{\left.\sum_{n \in \mathbb{Z}} c_{n}|n\rangle_{\delta}\left|\sum_{n} n^{2}\right| c_{n}\right|^{2}<\infty\right\} .
$$

Note that in each representation $\delta, \hat{p}$ has basis (2.8) as a complete set of eigenvectors:

$$
\hat{p}|n\rangle_{\delta}=(n+\delta) \hbar|n\rangle_{\delta} .
$$

Equation (2.10) shows that representations for different $\delta$ are in fact unitarily inequivalent. The parameter $\delta$ determines the fraction of $2 \pi$, the phase of a particle acquires, if it runs once 
round the circle. In periodic crystals, the parameter $\delta$ can be seen as the quasimomentum of the Bloch waves [2]. The meaning of the parameter $\delta$ will be illustrated later in more detail, when we derive the semiclassical propagator on the circle, which heavily depends on $\delta$, since it keeps track of different paths with different winding number, where the relative phase is crucial.

In [3], it was reasoned that the cases $\delta=0$ and $\delta=1 / 2$ are the most important ones, since in these representations the systems are invariant under time-reversal symmetry. In these representations one cannot distinguish between a wave running clockwise or anti-clockwise around the circle, since the first one acquires a phase of $\mathrm{e}^{2 \pi \mathrm{i} \delta}$ and the other one a phase of $\mathrm{e}^{-2 \pi \mathrm{i} \delta}$, which is not the same for $\delta \neq 0, \frac{1}{2}$. Still, one could imagine systems where nontrivial phase shifts occur due to complicated interactions, which explicitly distinguish between clockwise or anti-clockwise moving waves. In particular, the topic of fractional optical angular momentum $(\mathrm{OAM})$ is discussed in [2]. So in what follows, we will not fix $\delta \in[0,1)$ to keep the results as general as possible.

\section{Coherent states}

\subsection{Definition}

On the circle, as well as on any other compact, connected Lie group, one can define the 'complexifier coherent states' [7], which for the case of the circle are different for every $\delta$. They are furthermore labelled by a squeezing parameter $s>0$ and a complex number $z=\phi+\mathrm{i} p / \hbar$ :

$$
\begin{aligned}
|z\rangle_{\delta} & =\sum_{n \in \mathbb{Z}} \exp \left(-(n+\delta)^{2} \frac{s^{2}}{2}-\mathrm{i}(n+\delta) z\right)|n\rangle_{\delta} \\
& =\sum_{n \in \mathbb{Z}} \exp \left(-(n+\delta)^{2} \frac{s^{2}}{2}+(n+\delta) \frac{p}{\hbar}-\mathrm{i}(n+\delta) \phi\right)|n\rangle_{\delta} \\
& =\exp \left(-\delta^{2} \frac{s^{2}}{2}-\mathrm{i} \delta(z-\phi)\right) \vartheta_{3}\left(\frac{\phi-z}{2 \pi} \mid \mathrm{i} \frac{s^{2}}{2 \pi}\right)
\end{aligned}
$$

where

$$
\vartheta_{3}(z \mid \tau)=\sum_{n \in \mathbb{Z}} \exp \left(\pi \mathrm{i} n^{2} \tau+2 \pi \mathrm{i} n z\right)
$$

is the Jacobi-theta function of the third kind. These functions and their properties are well known (see, e.g., [34]) and serve as an analogue of the Gaussian curve for periodic functions. The coherent states as well as inner products between them can be expressed in these functions.

We will not use the form of the coherent states as Jacobi-theta functions, but will rather stay with the explicit form (3.1) as series over integer numbers. This form will prove to be better for the explicit manipulations we will perform in chapters 4 and 5 .

Note that the map $z \mapsto|z\rangle_{\delta}$ is periodic in the sense that

$$
|z+2 \pi\rangle_{\delta}=\mathrm{e}^{-2 \pi \mathrm{i} \delta}|z\rangle_{\delta}
$$

The states (3.1) are not normalized, but instead we have

$$
\left\|z_{\delta}\right\|^{2}:={ }_{\delta}\langle z \mid z\rangle_{\delta}=\sum_{n \in \mathbb{Z}} \exp \left(-(n+\delta)^{2} s^{2}+2(n+\delta) \frac{p}{\hbar}\right) .
$$


The infinite sum in (3.4) can be brought into a nicer form by the so-called 'Poisson summation formula' (see e.g. [30]), given by

$$
\sum_{n \in \mathbb{Z}} f(a n)=\frac{1}{a} \sum_{n \in \mathbb{Z}} \tilde{f}\left(\frac{2 \pi n}{a}\right),
$$

where $\tilde{f}$ is the Fourier transform of $f$ :

$$
\tilde{f}(k)=\int_{\mathbb{R}} \mathrm{d} x f(x) \mathrm{e}^{-\mathrm{i} k x} .
$$

Choosing $f(x)=\exp \left(-x^{2} s^{2}+2 x p / \hbar\right)$, with

$$
\tilde{f}(k)=\sqrt{\frac{\pi}{s^{2}}} \exp \left(\frac{p^{2}}{s^{2} \hbar^{2}}-\frac{k^{2}-4 \mathrm{i} k p / \hbar}{4 s^{2}}\right),
$$

equation (3.4) can be rewritten as

$$
\begin{aligned}
\left\|z_{\delta}\right\|^{2} & =\sqrt{\frac{\pi}{s^{2}}} \exp \left(\frac{p^{2}}{s^{2} \hbar^{2}}\right) \sum_{n \in \mathbb{Z}} \exp \left(-2 \pi n \delta \mathrm{i}-\frac{\pi^{2} n^{2}-\mathrm{i} \pi n p / \hbar}{s^{2}}\right) \\
& =\sqrt{\frac{\pi}{s^{2}}} \exp \left(\frac{p^{2}}{s^{2} \hbar^{2}}\right)\left[1+\sum_{n \neq 0}\left(-2 \pi n \delta \mathrm{i}-\frac{\pi^{2} n^{2}-\mathrm{i} \pi n p / \hbar}{s^{2}}\right)\right] .
\end{aligned}
$$

One can easily show the inequality

$$
\left|\sum_{n \neq 0} \exp \left(-2 \pi n \delta \mathrm{i}-\frac{\pi^{2} n^{2}-\mathrm{i} \pi n p / \hbar}{s^{2}}\right)\right| \leqslant 2 \frac{\mathrm{e}^{-\frac{\pi^{2}}{s^{2}}}}{1-\mathrm{e}^{-\frac{\pi^{2}}{s^{2}}}},
$$

which tends to zero faster than any power of $s$. Therefore, we denote it as $O\left(s^{\infty}\right)$ and write

$$
\left\|z_{\delta}\right\|^{2}=\sqrt{\frac{\pi}{s^{2}}} \exp \left(\frac{p^{2}}{s^{2} \hbar^{2}}\right)\left(1+O\left(s^{\infty}\right)\right) .
$$

The inner product between two coherent states $|z\rangle_{\delta}$ and $|w\rangle_{\delta}$ can be obtained by a similar calculation, using again the Poisson summation formula

$$
\begin{aligned}
{ }_{\delta}\left\langle z^{\prime} \mid z\right\rangle_{\delta}= & \sqrt{\frac{\pi}{s^{2}}} \sum_{n \in \mathbb{Z}} \exp \left[2 \pi \mathrm{i} n \delta-\left(n \pi-\frac{\bar{z}^{\prime}-z}{2}\right)^{2} \frac{1}{s^{2}}\right] \\
= & \sqrt{\frac{\pi}{s^{2}}} \exp \left[\left(\frac{p+p^{\prime}}{2 s \hbar}\right)^{2}\right] \sum_{n \in \mathbb{Z}} \exp \left[2 \pi \mathrm{i} n \delta-\left(\frac{\phi^{\prime}-\phi-2 \pi n}{2 s}\right)^{2}\right. \\
& \left.+2 \mathrm{i}\left(\frac{\phi^{\prime}-\phi-2 \pi n}{2 s}\right) \frac{p^{\prime}+p}{2 s \hbar}\right] .
\end{aligned}
$$

Note that this overlap can also be brought into the form of a Jacobi-theta function, in particular

$$
{ }_{\delta}\left\langle z^{\prime} \mid z\right\rangle_{\delta}=\sqrt{\frac{\pi}{s^{2}}} \exp \left(-\frac{\left(\bar{z}^{\prime}-z\right)^{2}}{4 s^{2}}\right) \vartheta_{3}\left(-\mathrm{i} \frac{\bar{z}^{\prime}-z}{2 s^{2}}+\delta \mid \frac{\mathrm{i}}{\pi s^{2}}\right) .
$$

From (3.11) and (3.10), we obtain the overlap of two coherent states as

$$
\begin{aligned}
\frac{{ }_{\delta}\left\langle z^{\prime} \mid z\right\rangle_{\delta}}{\left\|z_{\delta}^{\prime}\right\|\left\|z_{\delta}\right\|}=\exp & {\left[-\left(\frac{p^{\prime}-p}{2 s \hbar}\right)^{2}\right] \sum_{n \in \mathbb{Z}} \exp \left[2 \pi \mathrm{i} n \delta-\left(\frac{\phi^{\prime}-\phi-2 \pi n}{2 s}\right)^{2}\right.} \\
+ & \left.2 \mathrm{i}\left(\frac{\phi^{\prime}-\phi-2 \pi n}{2 s}\right) \frac{p^{\prime}+p}{2 s \hbar}\right]\left(1+O\left(s^{\infty}\right)\right) .
\end{aligned}
$$


From formula (3.13), one can see that coherent states $|z\rangle_{\delta}$ and $\left|z^{\prime}\right\rangle_{\delta}$ with $z \neq z^{\prime}$, i.e. states labelled by different points, $z=\phi+\mathrm{i} p / \hbar$ and $z^{\prime}=\phi^{\prime}+\mathrm{i} p^{\prime} / \hbar$, have an overlap that tends to zero faster than any power of $s$, so the overlap function (3.13) is peaked at $z=z^{\prime}$, the peak becoming sharper as $s \rightarrow 0$. If $\phi^{\prime} \neq \pm \pi$ and $s \ll \pi$, then all terms with $n \neq 0$ in the infinite sum (3.13) are of order $O\left(s^{\infty}\right)$, so we get

$\frac{{ }_{\delta}\left\langle z^{\prime} \mid z\right\rangle_{\delta}}{\left\|z_{\delta}^{\prime}\right\|\left\|z_{\delta}\right\|}=\exp \left[-\left(\frac{p^{\prime}-p}{2 s \hbar}\right)^{2}-\left(\frac{\phi^{\prime}-\phi}{2 s}\right)^{2}+2 \mathrm{i}\left(\frac{\phi^{\prime}-\phi}{2 s}\right) \frac{p^{\prime}+p}{2 s \hbar}\right]\left(1+O\left(s^{\infty}\right)\right)$.

In particular, the overlap for fixed $z^{\prime}$ is - up to small corrections in $s$-a Gaussian in the complex $z$-plane centred at $z=z^{\prime}$, with width $s$.

Note that (2.8) defines a $\phi$-representation via $\langle\phi \mid n\rangle_{\delta}=\exp [-\mathrm{i}(n+\delta) \phi]$. Using (3.5), one can show that in this representation the coherent states are infinite superpositions of Gaussian wavepackets with a width $s$, each translated by $\phi \rightarrow \phi+2 \pi n$, ensuring that the resulting function is $2 \pi$-periodic in $\phi$. This shows that the complexifier coherent states on the circle are the periodically continued harmonic oscillator coherent states, which have been used earlier $[15,19]$. As long as the width $s$ of these wavepackets is much smaller than the period $2 \pi$, the different Gaussians interfere little with each other, and one can restrict oneself to one Gaussian in calculations. But if the spreading $s$ exceeds, say, $\pi$, one has to take the infinite mutual interference of the Gaussians into account, which makes calculations quite difficult.

Since the parameter $s$ measures the spreading of the wavefunction $\psi_{z}(\phi)=\langle\phi \mid z\rangle_{\delta}$ compared to the circumference of the circle on which the system propagates, the complexifier coherent states only describe systems being 'close to classical point particles' if $s$ is small: $s \ll \pi$. This feature is quite natural, since, if a particle moves on a circle with a de Broglie wavelength the same order of magnitude as the circumference of the circle, one cannot expect this particle to behave classically. The wavefunction of the particle will interfere with itself 'around the circle', which is not possible in classical mechanics. This is why the states are called 'coherent' rather than 'semiclassical states', and is simply due to the compact topology of configuration space. The limit in which quantum mechanics on the real line is recovered is then performed as $s \rightarrow 0$.

\subsection{Properties}

The complexifier coherent states (3.1) have a number of properties they share with ordinary harmonic oscillator coherent states, which have been exhibited in $[2,3,11-13,21]$ and are listed for the sake of completeness.

- Reproduction of classical values. The expectation values of certain operators in the coherent states labelled by $z=\phi+\mathrm{i} p / \hbar$ agree-up to small corrections in $s$-with the value of the corresponding classical phase-space functions, evaluated at the points $\left(\phi, s^{-2} p\right)$. This will be demonstrated with the basic operators $\exp (\mathrm{i} \hat{\phi})$ and $\hat{p}$. We start with $\exp (\mathrm{i} \hat{\phi})$. For this we remember (2.6) and (2.8), in particular

$$
\exp (\mathrm{i} \hat{\phi})|n\rangle_{\delta}=|n+1\rangle_{\delta} \text {. }
$$

With $z=\phi+\mathrm{i} p / \hbar$ and the definition of the coherent states (3.1), we get

$$
{ }_{\delta}\langle z|\exp (\mathrm{i} \hat{\phi})| z\rangle_{\delta}=\sum_{n \in \mathbb{Z}} \exp \left(-(n+\delta)^{2} \frac{s^{2}}{2}-(n-1+\delta)^{2} \frac{s^{2}}{2}+(2 n+2 \delta-1) \frac{p}{\hbar}+\mathrm{i} \phi\right) \text {. }
$$


With the help of the Poisson summation formula (3.5), we obtain after some straightforward computation:

$$
\begin{aligned}
{ }_{\delta}\langle z|\exp (\mathrm{i} \hat{\phi})| z\rangle_{\delta} & =\sqrt{\frac{\pi}{s^{2}}} \exp \left(\mathrm{i} \phi-\frac{s^{2}}{4}+\frac{p^{2}}{s^{2} \hbar^{2}}\right) \\
& \times \sum_{n \in \mathbb{Z}} \exp \left(2 \pi n \delta \mathrm{i}-\frac{\pi^{2} n^{2}+\mathrm{i} n \pi p / \hbar-\mathrm{i} \pi n}{s^{2}}\right) .
\end{aligned}
$$

By using (3.10) and again estimating all terms with $n \neq 0$,

$$
\sum_{n \neq 0}\left(2 \pi n \delta \mathrm{i}-\frac{\pi^{2} n^{2}+\mathrm{i} n \pi p / \hbar-\mathrm{i} \pi n}{s^{2}}\right)=O\left(s^{\infty}\right),
$$

we arrive at the result

$$
\begin{aligned}
\frac{\delta_{\delta}\langle z|\exp (\mathrm{i} \hat{\phi})| z\rangle_{\delta}}{{ }_{\delta}\langle z \mid z\rangle_{\delta}} & =\exp \left(\mathrm{i} \phi-\frac{s^{2}}{4}\right)\left(1+O\left(s^{\infty}\right)\right) \\
& =\exp (\mathrm{i} \phi)\left(1+O\left(s^{2}\right)\right)
\end{aligned}
$$

So, up to order $O\left(s^{2}\right)$, the expectation value of $\exp (\mathrm{i} \hat{\phi})$ in coherent states labelled by the complex number $z=\phi+\mathrm{i} p / \hbar$ agrees with the value of the classical phase-space function $\exp (\mathrm{i} \phi)$ at this point. By a similar calculation, one can even show that the expectation value of the relative phase between two particles in coherent states labelled by $z, z^{\prime}$ is $\exp \left[\mathrm{i}\left(\phi-\phi^{\prime}\right)\right]$, up to $O\left(s^{\infty}\right)$ corrections.

We continue with $\hat{p}$. From (2.10) and (3.5), we get

$$
\begin{aligned}
\delta\langle z|\hat{p}| z\rangle_{\delta} & =\hbar \sum_{n \in \mathbb{Z}}(n+\delta) \exp \left(-(n+\delta)^{2} s^{2}+2(n+\delta) \frac{p}{\hbar}\right) \\
& =\sqrt{\frac{\pi}{s^{2}}} \exp \left(\frac{p^{2}}{s^{2} \hbar^{2}}\right) \sum_{n \in \mathbb{Z}}\left(\frac{p}{s^{2}}-\pi n \hbar\right) \exp \left(2 \pi n \delta \mathrm{i}-\frac{\pi^{2} n^{2}+\mathrm{i} p n / \hbar}{s^{2}}\right) .
\end{aligned}
$$

With this and (3.10) we obtain the result

$$
\frac{{ }_{\delta}\langle z|\hat{p}| z\rangle_{\delta}}{{ }_{\delta}\langle z \mid z\rangle_{\delta}}=\frac{p}{s^{2}}\left(1+O\left(s^{\infty}\right)\right) .
$$

So the coherent state labelled by the complex number $z=\phi+\mathrm{i} p / \hbar$ is peaked around the phase-space point $\left(\phi, s^{-2} p\right)$.

Note that the expectation values of the operators $\exp (\mathrm{i} \hat{\phi})$ and $\hat{p}$ give the classical values $\exp (\mathrm{i} \phi)$ and $s^{-2} p$, respectively, only up to corrections in $s$. If $s \rightarrow 0$ and $s^{-2} p$ is held fixed, the corrections go to zero, which corresponds to the radius of the circle going to infinity, suppressing the self-interference of the wavefunction. Quantum mechanics on the real line is recovered in that limit. In particular, the expectation values of the operators $\hat{X}$ and $\hat{P}$ in the coherent states $|z\rangle=|q+\mathrm{i} p\rangle$ on the real line reproduce the classical values $q$ and $p$ exactly.

- Resolution of the identity. Apart from reproducing classical phase-space function values, the coherent states form an overcomplete system of vectors (see section 4). With $z=\phi+\mathrm{i} p / \hbar$, we get

$$
\frac{1}{\sqrt{\pi} s \hbar} \int_{\mathbb{R}} \mathrm{d} p \int_{-\pi}^{\pi} \frac{\mathrm{d} \phi}{2 \pi} \exp \left(-\frac{p^{2}}{s^{2} \hbar^{2}}\right)|z\rangle_{\delta \delta}\langle z|=\mathbb{1},
$$

which can be shown easily by calculating the action of the left-hand side of (3.22) on a basis vector $|n\rangle_{\delta}$. 
- Ladder operator eigenstates. Both operators $\exp \left(-s^{2} \hat{p}^{2} / 2 \hbar^{2}\right)$ and $\exp (\mathrm{i} \hat{\phi})$ are bounded, so the operator

$$
\hat{g}=\exp \left(-s^{2} \frac{\hat{p}^{2}}{2 \hbar^{2}}\right) \exp (\mathrm{i} \hat{\phi}) \exp \left(s^{2} \frac{\hat{p}^{2}}{2 \hbar^{2}}\right)
$$

is well defined on the domain of definition of $\hat{p}$ (2.9). We have

$$
\hat{g}|n\rangle_{\delta}=\exp \left[\frac{s^{2}}{2}\left((n+\delta)^{2}-(n+\delta+1)^{2}\right)\right]|n+1\rangle_{\delta}
$$

With this one obtains

$$
\begin{aligned}
\hat{g}|z\rangle_{\delta} & =\sum_{n \in \mathbb{Z}} \exp \left(-(n+\delta)^{2} \frac{s^{2}}{2}-\mathrm{i}(n+\delta) z\right) \hat{g}|n\rangle_{\delta} \\
& =\sum_{n \in \mathbb{Z}} \exp \left(-(n+1+\delta)^{2} \frac{s^{2}}{2}-\mathrm{i}(n+\delta) z\right)|n+1\rangle_{\delta} \\
& =\mathrm{e}^{\mathrm{i} z}|z\rangle_{\delta} .
\end{aligned}
$$

So the coherent states labelled by $z$ are eigenvectors of $\hat{g}$ with eigenvalue $\mathrm{e}^{\mathrm{i} z}$. With (3.24) one can see that $\hat{g}$ and $\hat{g}^{\dagger}$ are in fact ladder operators, although their commutator is not proportional to unity. Note that, by formally using the Baker-Campbell-Hausdorff formula, one can bring $\hat{g}$ into the form of $\hat{g}=\exp \mathrm{i}(\hat{\phi}+\mathrm{i} \hat{p} / \hbar)$.

- Minimal uncertainty relationship. As demonstrated in [7] or [3], the fact that the coherent states are eigenvectors of $\hat{g}$ immediately implies that the coherent states saturate the Heisenberg inequality for the operators

$$
\hat{Q}=\frac{\hat{g}+\hat{g}^{\dagger}}{2}, \quad \hat{P}=\frac{\hat{g}-\hat{g}^{\dagger}}{2 i}
$$

that is

$$
\Delta_{z} \hat{Q} \Delta_{z} \hat{P}=\frac{1}{2}\langle[\hat{Q}, \hat{P}]\rangle_{z},
$$

where $\langle\cdot\rangle_{z}$ and $\Delta_{z}$ denote expectation value and standard deviation in $|z\rangle_{\delta}$, respectively.

\section{Bargmann-Segal representation and Hadamard decomposition}

In quantum mechanics on the real line, the harmonic oscillator coherent states $|z\rangle$ provide the Bargmann-Segal representation $\psi(z)=\langle\psi \mid z\rangle$ for Hilbert space states $\psi$. From this one can construct the Husimi distribution [20,31]

$$
\rho_{\text {Husimi }}(q, p)=\exp \left(-p^{2}-q^{2}\right)|\psi(q+\mathrm{i} p)|^{2} .
$$

This phase-space density provides a way to analyse dynamical properties of systems, in particular the behaviour of classically chaotic quantum systems $[20,32]$. It is possible to reconstruct the state $\psi$ from the zeros of its Husimi distribution and the specification of three complex numbers $C_{0}, C_{1}, C_{2}$ [32], which have to be derived from $\rho_{\text {Husimi }}$.

In the following, we will describe the analogous construction for the complexifier coherent states on the circle and show that the condition of periodicity in one phase-space variable poses significant limitations to the form of the Bargmann-Segal representation. In particular, the zeros of the Husimi distribution of a state determine this state apart from normalization and specification of an integer, which can be computed from $\rho_{\text {Husimi }}$. 
The Bargmann-Segal representation of a state $\psi$ on the circle, provided by the complexifier coherent states, is given by the function

$$
\psi_{\delta}(z)=\langle\psi \mid z\rangle_{\delta}=\sum_{n \in \mathbb{Z}} \exp \left(-(n+\delta)^{2} \frac{s^{2}}{2}-\mathrm{i}(n+\delta) z\right)\langle\psi \mid n\rangle_{\delta} .
$$

The map (4.2) is entire holomorphic. Note that (3.22) guarantees that

$$
\frac{1}{\sqrt{\pi} s \hbar} \int_{\mathbb{R}} \mathrm{d} p \int_{-\pi}^{\pi} \frac{\mathrm{d} \phi}{2 \pi} \exp \left(-\frac{p^{2}}{s^{2} \hbar^{2}}\right)\left|\psi_{\delta}(z)\right|^{2}=\langle\psi \mid \psi\rangle,
$$

which shows that the map between the vector $|\psi\rangle$ and the function $z \mapsto \psi_{\delta}(z)$ is an antiunitarity between the Hilbert space $L^{2}[0,2 \pi]$ and the Hilbert space $\mathcal{H} L^{2}\left(S^{1} \times \mathbb{R}, \mathrm{d} \mu\right)$. With the latter we mean the Hilbert space of periodic, holomorphic functions of $z=\phi+\mathrm{i} p / \hbar$ that are square integrable with respect to the measure

$$
\mathrm{d} \mu(z)=\frac{1}{\sqrt{\pi} s \hbar} \mathrm{d} p \frac{\mathrm{d} \phi}{2 \pi} \exp \left(-\frac{p^{2}}{s^{2} \hbar^{2}}\right) .
$$

That (4.2) is holomorphic has an important corollary. The set of coherent states $|z\rangle_{\delta}$ spans the Hilbert space, hence it is complete. It is even overcomplete in the sense that smaller subsets of it also span the Hilbert space. Let $\left\{z_{n}\right\}_{n \in \mathbb{N}}$ be a sequence of distinct complex numbers converging to $z \in \mathbb{C}$. Then the set of coherent states $\left\{|z\rangle_{\delta}\right\}_{n \in \mathbb{N}}$ is complete. This is quite easy to show by noting that, if $\psi$ is orthogonal to the span of the $\left|z_{n}\right\rangle_{\delta}$, then $\psi_{\delta}\left(z_{n}\right)=0$ for all $n$. But since $\psi_{\delta}(z)$ is holomorphic and the $z_{n}$ converge, then $\psi_{\delta} \equiv 0$ by the identity theorem for holomorphic functions. So the linear span of the $\left|z_{n}\right\rangle_{\delta}$ is dense in $L^{2}[0,2 \pi]$.

With this one can define the Husmi distribution of a state $\psi \in \mathcal{H}$ to be

$$
\rho_{\text {Husimi }}^{(\delta)}(\phi, p):=\frac{1}{\langle\psi \mid \psi\rangle}\left|\psi_{\delta}(\phi+\mathrm{i} p / \hbar)\right|^{2},
$$

in complete analogy to (4.1). Note that (4.5) is a special case of a broader class of phasespace (quasi)densities investigated in [21] for the case $\delta=0$, which also includes densities analogous to the Wigner- and the Glauber-Sudarshan functions.

Of particular interest are the zeros of the Husimi distribution (4.5), which coincide with the zeros of $\psi_{\delta}(z)$ (4.2). These functions will be investigated further. Just as in the case of harmonic oscillator coherent states, the holomorphic functions $\psi_{\delta}(z)$ are entire and of order at most 2 .

Proposition 4.1. Let $|\psi\rangle \in \mathcal{H}$. Then there exist constants $A, B>0$, such that

$$
\left|\psi_{\delta}(z)\right|=\left|\langle\psi \mid z\rangle_{\delta}\right| \leqslant A \exp \left(B|z|^{2}\right) .
$$

Proof. With $z=\phi+\mathrm{i} p / \hbar$, we get using the Poisson summation formula (3.5),

$$
\begin{aligned}
\left|\psi_{\delta}(z)\right| \exp \left(-\frac{|z|^{2}}{2 s^{2}}\right) & \leqslant\left|\psi_{\delta}(z)\right| \exp \left(-\frac{p^{2}}{2 s^{2} \hbar^{2}}\right) \\
& =\left|\sum_{n \in \mathbb{Z}} \exp \left(-(n+\delta)^{2} \frac{s^{2}}{2}-\mathrm{i}(n+\delta) z-\frac{p^{2}}{2 s^{2} \hbar^{2}}\right)\langle\psi \mid n\rangle_{\delta}\right| \\
& \leqslant \sum_{n \in \mathbb{Z}} \exp \left(-(n+\delta)^{2} \frac{s^{2}}{2}+(n+\delta) \frac{p}{\hbar}-\frac{p^{2}}{2 s^{2} \hbar^{2}}\right)\left|\langle\psi \mid n\rangle_{\delta}\right| \\
& \leqslant \sum_{n \in \mathbb{Z}} \exp \left[-\left(n+\delta-\frac{p}{s^{2} \hbar}\right)^{2} \frac{s^{2}}{2}\right] \psi_{\max }
\end{aligned}
$$




$$
\begin{aligned}
& =\psi_{\max } \sqrt{\frac{2 \pi}{s^{2}}} \sum_{n \in \mathbb{Z}} \mathrm{e}^{2 \pi \mathrm{i} n \delta} \exp \left(-\frac{2 \pi^{2} n^{2}+2 \pi n \mathrm{i} p / \hbar}{s^{2}}\right) \\
& \leqslant \psi_{\max } \sqrt{\frac{2 \pi}{s^{2}}} \sum_{n \in \mathbb{Z}} \exp \left(-\frac{2 \pi^{2} n^{2}}{s^{2}}\right),
\end{aligned}
$$

with $\psi_{\max }=\max \left\{\left|\langle\psi \mid n\rangle_{\delta}\right|, n \in \mathbb{Z}\right\}$, and therefore

$$
|\psi(z)| \leqslant \psi_{\max } \sqrt{\frac{2 \pi}{s^{2}}} \sum_{n \in \mathbb{Z}} \exp \left(-\frac{2 \pi^{2} n^{2}}{s^{2}}\right) \exp \left(\frac{|z|^{2}}{2 s^{2}}\right)
$$

what was to be shown.

In fact, 2 is the best estimate one can give for the order of the holomorphic functions $\psi_{\delta}(z)$. Although there are functions that are of order 1 (in particular $z \mapsto{ }_{\delta}\langle n \mid z\rangle_{\delta}$ ), there are also examples of functions of order 2 (for instance the one belonging to $|\psi\rangle=\sum_{n \neq 0} n^{-2}|n\rangle_{\delta}$ ).

Each entire holomorphic function can be expressed in terms of its zeros. This representation is called the Hadamard decomposition (see, e.g., [33]). In particular, for $\psi(z)$ being entire holomorphic and of order at most 2 , there are constants $m \in \mathbb{N}, C_{0}, C_{1}, C_{2} \in \mathbb{C}$ such that

$$
\psi(z)=z^{m} \mathrm{e}^{C_{0}+C_{1} z+C_{2} z^{2}} \prod_{n}\left(1-\frac{z}{z_{n}}\right) \exp \left[\frac{z}{z_{n}}+\frac{1}{2}\left(\frac{z}{z_{n}}\right)^{2}\right],
$$

where the $\left\{z_{n}\right\}$ are the zeros of $\psi(z)$.

The entire holomorphic functions $\psi_{\delta}(z)(4.2)$ have the following properties:

$$
\psi_{\delta}(z+2 \pi)=\mathrm{e}^{-2 \pi \mathrm{i} \delta} \psi_{\delta}(z)
$$

Therefore, their number of zeros can only be zero or infinity. On the other hand, one only has to know the zeros in the strip $[0,2 \pi) \times i \mathbb{R} \subset \mathbb{C}$ to know all zeros of $\psi(z)$.

Since $\psi_{\delta}$ is of order at most 2 (which relates to the growth behaviour), the sequence of its zeros $\left\{z_{n}\right\}_{n \in \mathbb{N}}$ is also of order at most 2. For sequences, this means that the number of sequence members contained in a circle of radius $R$ goes not faster than $R^{2}$, as $R$ grows large [33]. It follows that the sequence of zeros lying inside the strip $[0,2 \pi) \times \mathbb{i} \mathbb{R} \subset \mathbb{C}$ is of order at most 1 , which will be needed later.

Periodicity now restricts the possible values of $C_{1}, C_{2}$ in (4.9), as the following proposition shows.

Proposition 4.2. Let $\psi_{\delta}(z)$ be entire holomorphic in $z$, of order at most 2 and $\psi_{\delta}(z+2 \pi)=$ $\mathrm{e}^{-2 \pi \mathrm{i} \delta} \psi_{\delta}(z)$. Then there are constants $C \in \mathbb{C}$ and $m, l \in \mathbb{Z}$ such that

$\psi_{\delta}(z)=\mathrm{e}^{C+\mathrm{i}(l-\delta) z}\left[\sin \frac{z}{2} \exp \left(-\mathrm{i} \frac{z}{2}\right)\right]^{m} \prod_{k}\left[\frac{\sin \frac{z-a_{k}}{2}}{\sin \frac{-a_{k}}{2}} \exp \left(-v_{k} \mathrm{i} \frac{z}{2}\right)\right]$,

where $a_{k}$ are the zeros in the strip $[0,2 \pi) \times \mathrm{i} \mathbb{R} \subset \mathbb{C}$, apart from 0 , and $v_{k}:=\operatorname{sgn} \operatorname{Im} a_{k}$ the sign of the imaginary part of $a_{k}$.

Proof. We start with (4.9) and note that the zeros are $\left\{z_{k}+2 \pi n \mid n \in \mathbb{Z}\right\}$, because of (4.10). Then we divert the possible $m$-fold zeros at $2 \pi n$ : 


$$
\begin{aligned}
\psi_{\delta}(z)=\left[z \prod_{n \neq 0}\right. & \left.\left(1-\frac{z}{2 \pi n}\right) \exp \left(\frac{z}{2 \pi n}+\frac{1}{2}\left(\frac{z}{2 \pi n}\right)^{2}\right)\right]^{m} \\
& \times \mathrm{e}^{C_{0}+C_{1} z+C_{2} z^{2}} \prod_{k} \prod_{n \in \mathbb{Z}}\left(1-\frac{z}{a_{k}+2 \pi n}\right) \exp \left[\frac{z}{a_{k}+2 \pi n}+\frac{1}{2}\left(\frac{z}{a_{k}+2 \pi n}\right)^{2}\right] \\
= & {\left[z \prod_{n=1}^{\infty}\left(1-\frac{z^{2}}{4 \pi^{2} n^{2}}\right) \exp \left(\frac{1}{4 \pi^{2} n^{2}} z^{2}\right)\right]^{m} \mathrm{e}^{C_{0}+C_{1} z+C_{2} z^{2}} } \\
& \times \prod_{k}\left[\left(1-\frac{z}{a_{k}}\right) \prod_{n=1}^{\infty}\left(1-\frac{z}{a_{k}+2 \pi n}\right)\left(1-\frac{z}{a_{k}-2 \pi n}\right)\right. \\
& \left.\times \exp \left(\mathfrak{C}_{1}\left(a_{k}\right) z+\frac{1}{2} \mathfrak{C}_{2}\left(a_{k}\right) z^{2}\right)\right]
\end{aligned}
$$

Here $a_{k}$ are the zeros inside the strip $[0,2 \pi) \times \mathrm{i} \mathbb{R}$ apart from 0 , and

$\mathfrak{C}_{1}\left(a_{k}\right):=\frac{1}{a_{k}}+\sum_{n=1}^{\infty} \frac{2 a_{k}}{a_{k}^{2}-4 \pi^{2} n^{2}}=\frac{1}{a_{k}}+\frac{\Psi\left(1-\frac{a_{k}}{2 \pi}\right)-\Psi\left(1+\frac{a_{k}}{2 \pi}\right)}{2 \pi}=\frac{1}{2} \cot \frac{a_{k}}{2}$

$\mathfrak{C}_{2}\left(a_{k}\right):=\frac{1}{a_{k}^{2}}+\sum_{n=1}^{\infty} \frac{1}{\left(a_{k}+2 \pi n\right)^{2}}+\frac{1}{\left(a_{k}-2 \pi n\right)^{2}}=\frac{1}{a_{k}^{2}}+\frac{1}{4 \sin ^{2} \frac{a_{k}}{2}}$,

where $\Psi$ denotes the Digamma function $\Psi(z)=\Gamma^{\prime}(z) / \Gamma(z)$. Now we have to employ the identities

$$
\sin z=z \prod_{n=1}^{\infty}\left(1-\frac{z^{2}}{\pi^{2} n^{2}}\right)
$$

and

$\sin \left(\frac{z-a}{2}\right)=-\sin \left(\frac{a}{2}\right)\left(1-\frac{z}{a}\right) \prod_{n=1}^{\infty}\left(1-\frac{z}{a+2 \pi n}\right)\left(1-\frac{z}{a-2 \pi n}\right)$

for $\frac{a}{2 \pi} \notin \mathbb{Z}$. Equation (4.14) is well known, and (4.15) will be proved in the appendix section. Reinserting these two identities in (4.12), we obtain

$\psi_{\delta}(z)=\sin ^{m} \frac{z}{2} \exp \left(m z^{2} \sum_{n=1}^{\infty} \frac{1}{4 \pi^{2} n^{2}}\right) \mathrm{e}^{C_{0}+C_{1} z+C_{2} z^{2}} \prod_{k} \frac{\sin \frac{z-a_{k}}{2}}{\sin \frac{-a_{k}}{2}} \exp \left(\mathfrak{C}_{1}\left(a_{k}\right) z+\frac{1}{2} \mathfrak{C}_{2}\left(a_{k}\right) z^{2}\right)$.

The first exponential factor can be absorbed into a redefinition of $C_{2}$ :

$\psi_{\delta}(z)=\sin ^{m} \frac{z}{2} \mathrm{e}^{C_{0}+C_{1} z+\widetilde{C}_{2} z^{2}} \prod_{k} \frac{\sin \frac{z-a_{k}}{2}}{\sin \frac{-a_{k}}{2}} \exp \left(\mathfrak{C}_{1}\left(a_{k}\right) z+\frac{1}{2} \mathfrak{C}_{2}\left(a_{k}\right) z^{2}\right)$.

Periodicity now demands that

$$
\begin{aligned}
& \psi_{\delta}(z)=\mathrm{e}^{2 \pi \mathrm{i} \delta} \psi(z+2 \pi)=(-1)^{m} \sin \frac{z}{2} \mathrm{e}^{2 \pi \mathrm{i} \delta} \mathrm{e}^{C_{0}+C_{1}(z+2 \pi)+\widetilde{C_{2}}(z+2 \pi)^{2}} \\
& \times \prod_{k}\left[-\frac{\sin \frac{z-a_{k}}{2}}{\sin \frac{-a_{k}}{2}} \exp \left(\mathfrak{C}_{1}\left(a_{k}\right)(z+2 \pi)+\frac{1}{2} \mathfrak{C}_{2}\left(a_{k}\right)(z+2 \pi)^{2}\right)\right] \\
& =\psi_{\delta}(z)(-1)^{m} \mathrm{e}^{2 \pi \mathrm{i} \delta+2 \pi C_{1}+4 \pi^{2} \widetilde{C_{2}}+4 \pi \widetilde{C}_{2} z} \prod_{k}\left[-\mathrm{e}^{2 \pi \mathfrak{C}_{1}\left(a_{k}\right)+4 \pi^{2} \mathfrak{C}_{2}\left(a_{k}\right)+4 \pi \mathfrak{C}_{2}\left(a_{k}\right) z}\right]
\end{aligned}
$$


SO

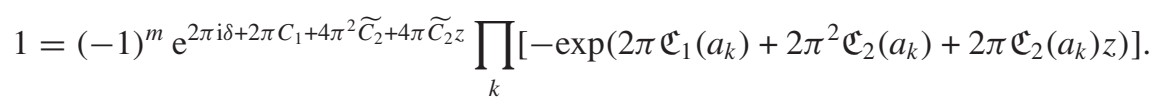

Note that this equation holds for all $z \in \mathbb{C}$, also for $z=0$. Hence, both

$$
\prod_{k}\left[-\exp \left(2 \pi \mathfrak{C}_{1}\left(a_{k}\right)+2 \pi^{2} \mathfrak{C}_{2}\left(a_{k}\right)+2 \pi \mathfrak{C}_{2}\left(a_{k}\right) z\right)\right]
$$

and

$$
\prod_{k}\left[-\exp \left(2 \pi \mathfrak{C}_{1}\left(a_{k}\right)+2 \pi^{2} \mathfrak{C}_{2}\left(a_{k}\right)\right)\right]
$$

converge. Thus, also their quotient

$$
\prod_{k}\left[\exp \left(2 \pi \mathfrak{C}_{2}\left(a_{k}\right) z\right)\right]=\exp \left(z \sum_{k} 2 \pi \mathfrak{C}_{2}\left(a_{k}\right)\right)
$$

converges for each $z \in \mathbb{C}$, and can be pulled out of the (possibly infinite) product in (4.19). So we get

$$
\begin{gathered}
1=(-1)^{m} \mathrm{e}^{2 \pi \mathrm{i} \delta+2 \pi C_{1}+4 \pi^{2} \tilde{C}_{2}} \exp \left(4 \pi \widetilde{C_{2}}+\sum_{k} 2 \pi \mathfrak{C}_{2}\left(a_{k}\right)\right) z \\
\times \prod_{k}\left[-\exp \left(2 \pi \mathfrak{C}_{1}\left(a_{k}\right)+2 \pi^{2} \mathfrak{C}_{2}\left(a_{k}\right)\right)\right] .
\end{gathered}
$$

This is only possible, if

$$
4 \pi \widetilde{C_{2}}+\sum_{k} 2 \pi \mathfrak{C}_{2}\left(a_{k}\right)=0
$$

as can be easily seen if one compares the growth behaviour on both sides of (4.23). Inserting (4.24) into (4.23) yields

$$
\mathrm{e}^{-\mathrm{i} m \pi-2 \pi \mathrm{i} \delta-2 \pi C_{1}}=\prod_{k}\left[-\exp \left(2 \pi \mathfrak{C}_{1}\left(a_{k}\right)\right)\right]=\prod_{k}\left[-\exp \left(\pi \cot \frac{a_{k}}{2}\right)\right] .
$$

To proceed, we need a technical lemma.

Lemma 4.1. Let $\left\{a_{k}\right\}$ be a sequence in $([0,2 \pi) \times \mathrm{i} \mathbb{R}) \backslash\{0\}$ of order 1 . Let $v_{k}:=\operatorname{sgn} \operatorname{Im} a_{k}$ be the sign of the imaginary part of $a_{k}($ with $\operatorname{sgn} 0:=1)$. Then

$$
\prod_{k}\left[-\exp \left(\pi \cot \frac{a_{k}}{2}\right)\right]=\exp \left[\sum_{k} \pi\left(\cot \frac{a_{k}}{2}+v_{k} \mathrm{i}\right)\right] \text {. }
$$

The proof of this lemma is rather technical and will be delivered in appendix B. With the help of (4.26), (4.25) can be rewritten. If the exponentials of two complex numbers are equal, the numbers themselves are equal up to a multiple of $2 \pi \mathrm{i}$. So, there is a number $l \in \mathbb{Z}$ such that

$$
-\mathrm{i} m \pi-2 \pi \mathrm{i} \delta-2 \pi C_{1}=2 \pi \sum_{k}\left(v_{k} \frac{\mathrm{i}}{2}+\mathfrak{C}_{1}\left(a_{k}\right)\right)+2 \pi \mathrm{i} l .
$$

Thus

$$
\mathrm{e}^{C_{1} z}=\mathrm{e}^{-\mathrm{i} \frac{m}{2} z} \mathrm{e}^{\mathrm{i}(l-\delta) z} \prod_{k} \exp \left[-z\left(v_{k} \frac{\mathrm{i}}{2}+\mathfrak{C}_{1}\left(a_{k}\right)\right)\right]
$$


Inserting (4.24) and (4.28) into (4.17) gives then

$\psi_{\delta}(z)=\mathrm{e}^{C+\mathrm{i}(l-\delta) z}\left[\sin \frac{z}{2} \exp \left(-\mathrm{i} \frac{z}{2}\right)\right]^{m} \prod_{k}\left[\frac{\sin \frac{z-a_{k}}{2}}{\sin \frac{-a_{k}}{2}} \exp \left(-v_{k} \mathrm{i} \frac{z}{2}\right)\right]$,

which completes the proof.

Note that the exponentials $\exp \left(-v_{k} \mathrm{i} \frac{z}{2}\right)$ in formula (4.29) can only be pulled out of the product if the set of zeros $a_{k}$ is finite. If this is not the case, the exponentials are needed for the infinite product to converge, of which the particular choice of the sign $v_{k}$ according to the imaginary part of $a_{k}$ takes care.

This proposition shows that a state is (up to normalization) completely determined by the zeros in its Hadamard decomposition and one further integer $l$. This is in contrast to the case of quantum mechanics on the real line, where instead of the integer $l$ one has to specify two complex numbers $C_{1}$ and $C_{2}$.

From this, one can immediately conclude that the only states, whose Husimi distributions are positive definite, are (up to normalization) the basis vectors (2.8). Let $|\psi\rangle$ and $|\tilde{\psi}\rangle$ be two states such that their Bargmann-Segal representations (4.2) have the same zeros (with the same multiplicities), i.e.

$$
\psi_{\delta}(z)=0 \Leftrightarrow \tilde{\psi}_{\delta}(z)=0 .
$$

By (4.11) this is the case if and only if $\tilde{\psi}_{\delta}(z)=\exp (C+\mathrm{i} l z) \psi_{\delta}(z)$ for some $l \in \mathbb{Z}$. With the action of the ladder operators $\hat{g}$ and $\hat{g}^{\dagger}$ (3.23), one can immediately conclude that, up to normalization,

$$
|\tilde{\psi}\rangle=\left(\hat{g}^{\dagger}\right)^{l}|\psi\rangle
$$

So, since the Bargmann-Segal representation of the basis vectors (2.8) has no zeros and the basis vectors are transformed into each other by the application of $\hat{g}^{\dagger}$, as one can easily compute, every state whose Bargmann-Segal representation has no zeros, is, up to normalization, a basis vector $|n\rangle_{\delta}, n \in \mathbb{Z}$. In particular, the Husimi distribution of every complexifier coherent state $|z\rangle_{\delta}$ has at least one zero, in contrast to the situation for quantum mechanics on the real line. Even more, since the Husimi distribution of a coherent state can, with the definition (3.1), be written in terms of the third Jacobian-theta function (3.2),

$$
\psi_{\delta}^{z^{\prime}, t}(z):={ }_{\delta}\left\langle z^{\prime} \mid z\right\rangle_{\delta}=\mathrm{e}^{-\delta^{2} s^{2}} \vartheta_{3}\left(\frac{2 \mathrm{i} s^{2} \delta+\bar{z}^{\prime}-z}{2 \pi}, \frac{\mathrm{i} s^{2}}{\pi}\right),
$$

and the zeros of the theta function $\vartheta_{3}(z, \tau)$ for fixed $\tau$ are known [34] to be

$$
z_{0}=\left(k+\frac{1}{2}\right)+\left(m+\frac{1}{2}\right) \tau \quad \text { for all } k, m \in \mathbb{Z},
$$

we immediately see that the Husimi distribution of the coherent states has even infinitely many zeros.

As we have seen by (4.11), one needs considerably less effort to reconstruct the state by the zeros of the Bargmann-Segal representation (and hence the Husimi distribution). Also, these zeros for states on the circle behave quite differently than the ones for states on the real line.

\section{Semiclassical propagator}

\subsection{Semiclassical approximation}

One of the most widely acknowledged properties of the harmonic oscillator coherent states is the fact that they provide a gateway to semiclassical analysis of quantum mechanical systems 
on the real line. In particular, they can be used to approximate the propagator between coherent states $K_{\mathbb{R}}\left(z_{F}, t_{F}, z_{I}, t_{I}\right)$ or position eigenstates $K_{\mathbb{R}}\left(x_{F}, t_{F}, x_{I}, t_{I}\right)$ (see, e.g. [22, 25-29] and references therein). In this section, we will show that the coherent states presented in the last sections can be used to calculate the propagator for quantum mechanical systems on the circle in a semiclassical approximation in the same way. Although technically more elaborate, there is not much conceptual difference between the following derivation and the one in [22]. This will demonstrate that the $U(1)$-complexifier coherent states are in fact useful for a semiclassical analysis. So, in what follows, we will compute the coherent state propagator

$$
K_{S^{1}}\left(z_{F}, \tau, z_{I}, 0\right)={ }_{\delta}\left\langle z_{F}\left|\mathrm{e}^{-\frac{i}{\hbar} \hat{H} \tau}\right| z_{I}\right\rangle_{\delta}
$$

since the propagators in the 'angle', momentum or any mixed representation can be derived from it.

Although we are working with the semiclassical approximation of the propagator, an exact expression can be written down for the particular case of the freely moving particle on the circle. In 'angle' representation this free propagator has already been calculated in [23]. As a propagator between coherent states on the circle, this has been done and investigated in $[2,24]$.

In what follows, we will perform the analysis not only for the case of the free particle, but for arbitrary Hamiltonians, and will obtain the propagator in the semiclassical approximation.

We start with expanding the exponential. For large $N$, we have

$$
{ }_{\delta}\left\langle z_{F}\left|\mathrm{e}^{-\frac{\mathrm{i}}{\hbar} \hat{H} \tau}\right| z_{I}\right\rangle_{\delta} \approx_{\delta}\left\langle z_{F}\left|\left(\mathbb{1}-\mathrm{i} \frac{\tau}{N \hbar} \hat{H}\right)^{N}\right| z_{I}\right\rangle_{\delta} .
$$

Using the completeness of the coherent states (3.22), we get

$$
\begin{aligned}
{ }_{\delta}\left\langle z_{F}\left|\mathrm{e}^{-\frac{\mathrm{i}}{\hbar} \hat{H} \tau}\right| z_{I}\right\rangle_{\delta} & \approx \frac{1}{\sqrt{\pi s^{2} \hbar^{2}}} \int_{\mathbb{R}^{N+1}} \mathrm{~d} p_{N} \ldots \mathrm{d} p_{0} \int_{[0,2 \pi]^{N+1}} \frac{\mathrm{d} \phi_{N}}{2 \pi} \cdots \frac{\mathrm{d} \phi_{0}}{2 \pi} \\
& \times \prod_{k=0}^{N}\left[\exp \left(\frac{z_{k}-\bar{z}_{k}}{2 s}\right)^{2}\right] \delta\left\langle z_{F} \mid z_{N}\right\rangle_{\delta \delta}\left\langle z_{0} \mid z_{I}\right\rangle_{\delta} \\
& \times \prod_{k=0}^{N-1}\left[\delta\left\langle z_{k+1} \mid z_{k}\right\rangle_{d}\left(\mathbb{1}-\frac{\mathrm{i} \tau}{N \hbar} \mathcal{H}\left(\bar{z}_{k+1}, z_{k}\right)\right)\right],
\end{aligned}
$$

where the function $\mathcal{H}$ defined by

$$
\mathcal{H}(w, z):=\frac{{ }_{\delta}\langle\bar{w}|\hat{H}| z\rangle_{\delta}}{{ }_{\delta}\langle\bar{w} \mid z\rangle_{\delta}}
$$

is holomorphic in both variables.

With (3.11) we get

$$
\begin{aligned}
\delta_{\delta}\left\langle z_{F}\left|\mathrm{e}^{-\frac{\mathrm{i}}{\hbar} \hat{H} \tau}\right| z_{I}\right\rangle_{\delta} & \approx \frac{1}{\left(s^{2} \hbar\right)^{N+1}} \sqrt{\frac{\pi}{s^{2}}} \int_{\mathbb{R}^{N+1}} \mathrm{~d} p_{N} \ldots \mathrm{d} p_{0} \int_{[0,2 \pi]^{N+1}} \frac{\mathrm{d} \phi_{N}}{2 \pi} \ldots \frac{\mathrm{d} \phi_{0}}{2 \pi} \\
& \times \prod_{k=0}^{N}\left[\exp \left(\frac{z_{k}-\bar{z}_{k}}{2 s}\right)^{2}\right] \sum_{n \in \mathbb{Z}} \exp \left[2 \pi \mathrm{i} n \delta-\left(\frac{\bar{z}_{F}-z_{N}-2 \pi n}{2 s}\right)^{2}\right] \\
& \times \sum_{n \in \mathbb{Z}} \exp \left[2 \pi \mathrm{i} n \delta-\left(\frac{\bar{z}_{0}-z_{I}-2 \pi n}{2 s}\right)^{2}\right] \\
& \times \prod_{k=0}^{N-1} \sum_{n \in \mathbb{Z}} \exp \left[2 \pi \mathrm{i} n \delta-\left(\pi n-\frac{\bar{z}_{k+1}-z_{k}-2 \pi n}{2 s}\right)^{2}-\frac{\mathrm{i} \tau}{N \hbar} \mathcal{H}\left(\bar{z}_{k+1}, z_{k}\right)\right],
\end{aligned}
$$


where

$$
\left(\mathbb{1}-\frac{\mathrm{i} \tau}{N \hbar} \mathcal{H}\left(\bar{z}_{k+1}, z_{k}\right)\right) \approx \exp \left(-\frac{\mathrm{i} \tau}{N \hbar} \mathcal{H}\left(\bar{z}_{k+1}, z_{k}\right)\right)
$$

has been used. With

$$
\prod_{k=0}^{N-1}\left(\sum_{n \in \mathbb{Z}} f(n)\right)=\sum_{n_{1} \in \mathbb{Z}} \cdots \sum_{n_{N} \in \mathbb{Z}} \prod_{k=0}^{N-1} f\left(n_{k+1}\right),
$$

formula (5.5) can be rewritten as follows:

$$
\begin{aligned}
{ }_{\delta}\left\langle z_{F}\left|\mathrm{e}^{-\frac{\mathrm{i}}{\hbar} \hat{H} \tau}\right| z_{I}\right\rangle_{\delta} & \approx \frac{1}{\left(s^{2} \hbar\right)^{N+1}} \sqrt{\frac{\pi}{s^{2}}} \int_{\mathbb{R}^{N+1}} \mathrm{~d} p_{N} \ldots \mathrm{d} p_{0} \int_{[0,2 \pi]^{N+1}} \frac{\mathrm{d} \phi_{N}}{2 \pi} \cdots \frac{\mathrm{d} \phi_{0}}{2 \pi} \\
& \times \sum_{n_{0} \in \mathbb{Z}} \sum_{n_{1} \in \mathbb{Z}} \cdots \sum_{n_{N+1} \in \mathbb{Z}} \exp \left[f^{(\vec{n})}(\overrightarrow{\bar{z}}, \vec{z})\right]
\end{aligned}
$$

where

$$
f^{(\vec{n})}(\overrightarrow{\bar{z}}, \vec{z})=f^{\left(n_{0}, \ldots, n_{N+1}\right)}\left(\bar{z}_{0}, \ldots, \bar{z}_{N}, z_{0}, \ldots, z_{N}\right)
$$

is given by

$$
\begin{aligned}
f^{\left(n_{0}, \ldots, n_{N+1}\right)}\left(\bar{z}_{0}, \ldots, \bar{z}_{N}, z_{0}, \ldots, z_{N}\right)=2 \pi \mathrm{i} \delta\left(\sum_{k=0}^{N+1} n_{k}\right)+\sum_{k=0}^{N}\left(\frac{z_{k}-\bar{z}_{k}}{2 s}\right)^{2} \\
-\left(\frac{\bar{z}_{0}-z_{I}-2 \pi n_{0}}{2 s}\right)^{2}-\sum_{k=0}^{N-1}\left(\frac{\bar{z}_{k+1}-z_{k}-2 \pi n_{k+1}}{2 s}\right)^{2} \\
-\left(\frac{\bar{z}_{F}-z_{N}-2 \pi n_{N+1}}{2 s}\right)^{2}-\frac{\mathrm{i} \tau}{N \hbar} \sum_{k=0}^{N-1} \mathcal{H}\left(\bar{z}_{k+1}, z_{k}\right) .
\end{aligned}
$$

This formula can be simplified considerably by using the following property of $f^{(\vec{n})}(\overrightarrow{\bar{z}}, \vec{z})$ :

$$
\begin{array}{r}
f^{\left(n_{0}, \ldots, n_{N+1}\right)}\left(\bar{z}_{0}, \ldots, \bar{z}_{k}+2 \pi, \ldots, \bar{z}_{N}, z_{0}, \ldots, z_{k}+2 \pi, \ldots, z_{N}\right) \\
\quad=f^{\left(n_{0}, \ldots, n_{k}-1, n_{k+1}+1, \ldots, n_{N+1}\right)}\left(\bar{z}_{0}, \ldots, \bar{z}_{N}, z_{0}, \ldots, z_{N}\right),
\end{array}
$$

which can be readily seen from the explicit form $(5.10)$ of $f^{(\vec{n})}(\overrightarrow{\bar{z}}, \vec{z})$ and the fact that $\mathcal{H}$ is periodic in both variables (5.4), since the coherent states are.

We then have

$$
\begin{aligned}
\sum_{n_{0} \in \mathbb{Z}} \sum_{n_{1} \in \mathbb{Z}} \int_{[0,2 \pi]^{2}} \frac{\mathrm{d} \phi_{1}}{2 \pi} \frac{\mathrm{d} \phi_{0}}{2 \pi} \exp \left[f^{\left(n_{0}, \ldots, n_{N+1}\right)}\left(\bar{z}_{0}, \ldots, \bar{z}_{n}, z_{0}, \ldots, z_{N}\right)\right] \\
\quad=\sum_{n_{0} \in \mathbb{Z}} \sum_{n_{1} \in \mathbb{Z}} \int_{[0,2 \pi]^{2}} \frac{\mathrm{d} \phi_{1}}{2 \pi} \frac{\mathrm{d} \phi_{0}}{2 \pi} \exp \left[f^{\left(0, n_{1}-n_{0}, \ldots, n_{N+1}\right)}\left(\bar{z}_{0}+2 \pi n_{0}, \ldots, \bar{z}_{n}, z_{0}+2 \pi n_{0}, \ldots, z_{N}\right)\right] \\
=\sum_{n_{0} \in \mathbb{Z}} \sum_{n_{1} \in \mathbb{Z}} \int_{[0,2 \pi]^{2}} \frac{\mathrm{d} \phi_{1}}{2 \pi} \frac{\mathrm{d} \phi_{0}}{2 \pi} \exp \left[f^{\left(0, n_{1}, \ldots, n_{N+1}\right)}\left(\bar{z}_{0}+2 \pi n_{0}, \ldots, \bar{z}_{n}, z_{0}+2 \pi n_{0}, \ldots, z_{N}\right)\right] \\
=\sum_{n_{1} \in \mathbb{Z}} \int_{[0,2 \pi]} \frac{\mathrm{d} \phi_{1}}{2 \pi} \int_{\mathbb{R}} \frac{\mathrm{d} \phi_{0}}{2 \pi} \exp \left[f^{\left(0, n_{1} \ldots, n_{N+1}\right)}\left(\bar{z}_{0}, \ldots, \bar{z}_{n}, \ldots, z_{N}\right)\right] .
\end{aligned}
$$

In the second step, we have used the invariance under shifting the summation index $n_{1}$. The trick used in (5.12) can now be carried on over all $\phi_{k}$ to transform the integrations over 
$[0,2 \pi]$ into integrations over $\mathbb{R}$ while getting rid of the summations. The last summation over $n_{N+1}$, however, cannot be eliminated this way, and one obtains

$$
\begin{aligned}
{ }_{\delta}\left\langle z_{F}\left|\mathrm{e}^{-\frac{\mathrm{i}}{\hbar} \hat{H} \tau}\right| z_{I}\right\rangle_{\delta} & \approx \frac{1}{\left(s^{2} \hbar\right)^{N+1}} \sqrt{\frac{\pi}{s^{2}}} \sum_{n \in \mathbb{Z}} \int_{\mathbb{R}^{N+1}} \mathrm{~d} p_{N} \ldots \mathrm{d} p_{0} \int_{\mathbb{R}^{N+1}} \frac{\mathrm{d} \phi_{N}}{2 \pi} \cdots \frac{\mathrm{d} \phi_{0}}{2 \pi} \\
\times & \exp \left[f^{(0,0, \ldots, 0, n)}(\overrightarrow{\bar{z}}, \vec{z})\right] .
\end{aligned}
$$

The integrand now takes a much simpler form

$$
\begin{aligned}
f^{(0,0, \ldots, n)}(\overrightarrow{\bar{z}}, \vec{z}) & =2 \pi \mathrm{i} n \delta+\sum_{k=0}^{N}\left(\frac{z_{k}-\bar{z}_{k}}{2 s}\right)^{2}-\left(\frac{\bar{z}_{0}-z_{I}}{2 s}\right)^{2} \\
& -\sum_{k=0}^{N-1}\left(\frac{\bar{z}_{k+1}-z_{k}}{2 s}\right)^{2}-\left(\frac{\bar{z}_{F}-z_{N}-2 \pi n}{2 s}\right)^{2}-\frac{\mathrm{i} \tau}{N \hbar} \sum_{k=0}^{N-1} \mathcal{H}\left(\bar{z}_{k+1}, z_{k}\right) .
\end{aligned}
$$

We are now able to perform the semiclassical approximation. It assumes that the main part of the integral (5.13) comes from the stationary points of the integrand. These points are characterized by the condition that the first derivative of (5.14) vanishes:

$$
\begin{array}{ll}
0=\frac{\partial f^{(\overrightarrow{0}, n)}}{\partial z_{k}}=\frac{\bar{z}_{k+1}-\bar{z}_{k}}{2 s^{2}}-\frac{\tau}{N} \frac{\mathrm{i}}{\hbar} \partial_{2} \mathcal{H}\left(\bar{z}_{k+1}, z_{k}\right), \quad k=0, \ldots, N-1 \\
0=\frac{\partial f^{(\overrightarrow{0}, n)}}{\partial z_{N}}=\frac{2 \pi n-\bar{z}_{F}+\bar{z}_{N}}{2 s^{2}} \\
0=\frac{\partial f^{(\overrightarrow{0}, n)}}{\partial \bar{z}_{k+1}}=\frac{z_{k}-z_{k+1}}{2 s^{2}}-\frac{\tau}{N} \frac{\mathrm{i}}{\hbar} \partial_{1} \mathcal{H}\left(\bar{z}_{k+1}, z_{k}\right), \quad k=0, \ldots, N-1 \\
0=\frac{\partial f^{(0, n)}}{\partial \bar{z}_{0}}=\frac{z_{I}-z_{0}}{2 s^{2}},
\end{array}
$$

where we have used the notation

$$
\partial_{1} \mathcal{H}(w, z)=\frac{\partial \mathcal{H}}{\partial w}(w, z), \quad \partial_{2} \mathcal{H}(w, z)=\frac{\partial \mathcal{H}}{\partial z}(w, z)
$$

Let the points where $(5.15)$ are satisfied be called $(\overrightarrow{\vec{w}}, \vec{w})$.

Note that there may be more than one set of complex numbers satisfying (5.15) for every $N$ and $n$. This resembles the fact that there may be more than one classical trajectory from one point on the circle to another with the fixed winding number. We will keep this in mind, but refrain from introducing a special notation to keep track of the different paths, in order not to overburden the formulae.

We now expand $f^{(\overrightarrow{0}, n)}$ up to second order:

$$
\begin{aligned}
f^{(\overrightarrow{0}, n)}(\overrightarrow{\bar{z}}, \vec{z}) \approx & f^{(\overrightarrow{0}, n)}(\overrightarrow{\bar{w}}, \vec{w})+\frac{1}{2} \frac{\partial^{2} f^{(\overrightarrow{0}, n)}}{\partial z_{k} \partial z_{l}}(\overrightarrow{\bar{w}}, \vec{w}) z^{k} z^{l} \\
& +\frac{\partial^{2} f^{(\overrightarrow{0}, n)}}{\partial z_{k} \partial \bar{z}_{l}}(\overrightarrow{\bar{w}}, \vec{w}) z^{k} \bar{z}^{l}+\frac{1}{2} \frac{\partial^{2} f^{(\overrightarrow{0}, n)}}{\partial \bar{z}_{k} \partial \bar{z}_{l}}(\overrightarrow{\bar{w}}, \vec{w}) \bar{z}^{k} \bar{z}^{l}
\end{aligned}
$$

Inserting (5.17) into (5.13) enables us to evaluate the integral in (5.13) explicitly, for we will have to deal with ordinary Gaussian integrals only. From (5.15), we can compute the second derivatives of $f^{(\overrightarrow{0}, n)}$ : 


$$
\begin{aligned}
& \frac{\partial^{2} f^{(\overrightarrow{0}, n)}}{\partial z_{k} \partial z_{l}}=-\frac{\tau}{N} \frac{\mathrm{i}}{\hbar} \partial_{2}^{2} \mathcal{H}\left(\bar{z}_{k+1}, z_{k}\right) \delta_{k l} \quad k, l=0, \ldots, N-1, \\
& \frac{\partial^{2} f^{(\overrightarrow{0}, n)}}{\partial z_{N} \partial z_{k}}=0 \quad k=0, \ldots, N \\
& \frac{\partial^{2} f^{(\overrightarrow{0}, n)}}{\partial z_{k} \partial \bar{z}_{l+1}}=-\frac{1}{2 s^{2}} \delta_{k, l+1}+\left(\frac{1}{2 s^{2}}-\frac{\tau}{N} \frac{\mathrm{i}}{\hbar} \partial_{1} \partial_{2} \mathcal{H}\left(\bar{z}_{k+1}, z_{k}\right)\right) \delta_{k l}, \quad k, l=0, \ldots, N-1, \\
& \frac{\partial^{2} f^{\left(\overrightarrow{0}_{n}, n\right)}}{\partial z_{k} \partial \bar{z}_{0}}=-\frac{1}{2 s^{2}} \delta_{0 k} \quad k=0, \ldots, N, \\
& \frac{\partial^{2} f^{(\overrightarrow{0}, n)}}{\partial z_{N} \partial \bar{z}_{k}}=-\frac{1}{2 s^{2}} \delta_{0 N} \quad k=0, \ldots, N, \\
& \frac{\partial^{2} f^{(\overrightarrow{0}, n)}}{\partial \bar{z}_{k+1} \partial \bar{z}_{l+1}}=-\frac{\tau}{N} \frac{\mathrm{i}}{\hbar} \partial_{1}^{2} \mathcal{H}\left(\bar{z}_{k+1}, z_{k}\right) \delta_{k l} \quad k, l=0, \ldots, N-1, \\
& \frac{\partial^{2} f^{(0, n)}}{\partial \bar{z}_{0} \partial \bar{z}_{k}}=0 \quad k=0, \ldots, N
\end{aligned}
$$

Inserting (5.17) and (5.18) into (5.13), we obtain

$$
\begin{aligned}
{ }_{\delta}\left\langle z_{F}\left|\mathrm{e}^{-\frac{\mathrm{i}}{\hbar} \hat{H} \tau}\right| z_{I}\right\rangle_{\delta} \approx & \sqrt{\frac{\pi}{s^{2}}} \frac{1}{\left(\hbar s^{2}\right)^{N+1}} \sum_{n \in \mathbb{Z}} \mathrm{e}^{f^{(\overrightarrow{0}, n)}(\overrightarrow{\bar{w}}, \vec{w})} \int_{\mathbb{R}^{N+1}} \mathrm{~d} p_{N} \ldots \mathrm{d} p_{0} \int_{\mathbb{R}^{N+1}} \frac{\mathrm{d} \phi_{N}}{2 \pi} \cdots \frac{\mathrm{d} \phi_{0}}{2 \pi} \\
& \times \exp \left[-\frac{1}{2} \sum_{k=0}^{N-1} \frac{\tau}{N} \frac{\mathrm{i}}{\hbar} \partial_{2}^{2} \mathcal{H}\left(\bar{w}_{k+1}, w_{k}\right) z_{k}^{2}-\frac{1}{2 s^{2}} \sum_{k=0}^{N} z_{k} \bar{z}_{k}\right. \\
& +\sum_{k=0}^{N-1}\left(\frac{1}{2 s^{2}}-\frac{\tau}{N} \frac{\mathrm{i}}{\hbar} \partial_{1} \partial_{2} \mathcal{H}\left(\bar{w}_{k+1}, w_{k}\right)\right) z_{k} \bar{z}_{k+1} \\
& \left.-\frac{1}{2} \sum_{k=0}^{N-1} \frac{\tau}{N} \frac{\mathrm{i}}{\hbar} \partial_{1}^{2} \mathcal{H}\left(\bar{w}_{k+1}, w_{k}\right) \bar{z}_{k+1}^{2}\right] .
\end{aligned}
$$

For $z=\phi+\mathrm{i} p / \hbar$, the Gaussian integral

$\frac{1}{2 \pi \hbar} \int_{\mathbb{R}^{2}} \mathrm{~d} \phi \mathrm{d} p \exp \left(a_{1} z^{2}+a_{2} \bar{z}^{2}+a_{3} \bar{z} z+b_{1} z+b_{2} \bar{z}\right)$

$$
=\frac{1}{2} \frac{1}{\sqrt{a_{3}^{2}-4 a_{1} a_{2}}} \exp \left(\frac{a_{1} b_{2}^{2}+a_{2} b_{1}^{2}-a_{3} b_{1} b_{2}}{a_{3}^{2}-4 a_{1} a_{2}}\right)
$$

is used to integrate successively over $\mathrm{d} \phi_{k} \mathrm{~d} p_{k}$, starting with $k=0$. From (5.19), we can read off the parameters for this integration:

$$
\begin{aligned}
& a_{1}=-\frac{1}{2} \frac{\tau}{N} \frac{\mathrm{i}}{\hbar} \partial_{2}^{2} \mathcal{H}\left(\bar{w}_{1}, w_{0}\right), \quad a_{2}=0=: X_{0}, \quad a_{3}=-\frac{1}{2 s^{2}}, \\
& b_{1}=\left(\frac{1}{2 s^{2}}-\frac{\tau}{N} \frac{\mathrm{i}}{\hbar} \partial_{1} \partial_{2} \mathcal{H}\left(\bar{w}_{1}, w_{0}\right)\right) \bar{z}_{1}, \quad b_{2}=0 .
\end{aligned}
$$

The integration yields (keeping $X_{0}=0$ )

$$
\begin{aligned}
& { }_{\delta}\left\langle z_{F}\left|\mathrm{e}^{-\frac{\mathrm{i}}{\hbar} \hat{H} \tau}\right| z_{I}\right\rangle_{\delta} \approx \sqrt{\frac{\pi}{s^{2}}} \frac{1}{\left(\hbar s^{2}\right)^{N+1}} \mathrm{e}^{f^{(\overrightarrow{0}, n)}(\overrightarrow{\bar{w}}, \vec{w})} \int_{\mathbb{R}^{N}} \mathrm{~d} p_{N} \ldots \mathrm{d} p_{1} \int_{\mathbb{R}^{N}} \frac{\mathrm{d} \phi_{N}}{2 \pi} \cdots \frac{\mathrm{d} \phi_{1}}{2 \pi} \\
& \times \frac{1}{\sqrt{1+8 s^{4} \frac{\tau}{N} \frac{1}{\hbar} \partial_{2}^{2} H\left(\bar{w}_{1}, w_{0}\right) X_{0}}} \exp \left[\frac{\left(1-2 s^{2} \frac{\tau}{N} \frac{i}{\hbar} \partial_{1} \partial_{2} \mathcal{H}\left(\bar{w}_{1}, w_{0}\right)\right)^{2} X_{0}}{1+8 s^{4} \frac{\tau}{N} \frac{i}{\hbar} \partial_{2}^{2} H\left(\bar{w}_{1}, w_{0}\right) X_{0}}\right]
\end{aligned}
$$




$$
\begin{aligned}
& \times \exp \left[-\frac{1}{4 s^{2}} z_{N}^{2}-\frac{1}{2} \sum_{k=1}^{N-1} \frac{\tau}{N} \frac{\mathrm{i}}{\hbar} \partial_{2}^{2} \mathcal{H}\left(\bar{w}_{k+1}, w_{k}\right) z_{k}^{2}-\frac{1}{2 s^{2}} \sum_{k=1}^{N} z_{k} \bar{z}_{k}\right. \\
& +\sum_{k=1}^{N-1}\left(\frac{1}{2 s^{2}}-\frac{\tau}{N} \frac{\mathrm{i}}{\hbar} \partial_{1} \partial_{2} \mathcal{H}\left(\bar{w}_{k+1}, w_{k}\right) z_{k} \bar{z}_{k+1}\right) \\
& \left.-\frac{1}{2} \sum_{k=0}^{N-1} \frac{\tau}{N} \frac{\mathrm{i}}{\hbar} \partial_{1}^{2} \mathcal{H}\left(\bar{w}_{k+1}, w_{k}\right) \bar{z}_{k+1}^{2}\right] .
\end{aligned}
$$

Now one can read off the next set of parameters $a_{1}, a_{2}, a_{3}, b_{1}$ and $b_{2}$ to perform the subsequent integration over $\mathrm{d} \phi_{1} \mathrm{~d} p_{1}$ according to (5.20). The final result is

${ }_{\delta}\left\langle z_{F}\left|\mathrm{e}^{-\frac{i}{\hbar} \hat{H} \tau}\right| z_{I}\right\rangle_{\delta} \approx \sqrt{\frac{\pi}{s^{2}}} \sum_{n \in \mathbb{Z}} \mathrm{e}^{f^{(\overrightarrow{0}, n)}(\vec{w}, \vec{w})} \prod_{k=0}^{N-1} \frac{1}{\sqrt{1+8 s^{4} \frac{\tau}{N} \frac{1}{\hbar} \partial_{2}^{2} \mathcal{H}\left(\bar{w}_{k+1}, w_{k}\right) X_{k}}}$,

where $X_{k}$ are determined by the following recursion relation:

$X_{0}=0$

$X_{k+1}=\left(\frac{\left(1-2 s^{2} \frac{\tau}{N} \frac{\mathrm{i}}{\hbar} \partial_{1} \partial_{2} \mathcal{H}\left(\bar{w}_{k+1}, w_{k}\right)\right)^{2} X_{k}}{1+8 s^{4} \frac{\tau}{N} \frac{\mathrm{i}}{\hbar} \partial_{2}^{2} H\left(\bar{w}_{k+1}, w_{k}\right) X_{k}}\right)-\frac{1}{2} \frac{\tau}{N} \frac{\mathrm{i}}{\hbar} \partial_{1}^{2} \mathcal{H}\left(\bar{w}_{k+1}, w_{k}\right)$.

\subsection{The continuum limit}

We now perform the limit $N \rightarrow \infty$ in (5.23). The sequences $\bar{w}_{k}, w_{k}$ become functions $\bar{w}(t), w(t)$ with $t$ ranging from 0 to $\tau$ and conditions (5.15) turn into the Hamiltonian equations

$$
\begin{array}{rlrl}
\dot{w} & =-2 s^{2} \frac{\mathrm{i}}{\hbar} \partial_{1} \mathcal{H}(\bar{w}, w), & & w(0)=z_{I} \\
\dot{\bar{w}}=2 s^{2} \frac{\mathrm{i}}{\hbar} \partial_{2} \mathcal{H}(\bar{w}, w), & \bar{w}(\tau)=\bar{z}_{F}-2 \pi n .
\end{array}
$$

At this point, we encounter the same phenomenon that occurs in the case of the coherent state propagator of ordinary quantum mechanics. Equations (5.25) are the Hamiltonian equations for the system with the Hamiltonian $\mathcal{H}$, and the boundary conditions fix the starting point $z_{I}$ and the endpoint $z_{F}-2 \pi n$ in phase space. But the trajectory is already fixed by initial condition, and a solution $w(t)$ starting at $w(0)=z_{I}$ will most likely never go through $w(\tau)=z_{F}-2 \pi n$. The solution to this problem is as follows. One has to give up the condition that $w(t)$ and $\bar{w}(t)$ are to be complex conjugate to each other. Defining new variables

$$
u:=w, \quad v:=\bar{w},
$$

one can see that the integral (5.13) is complex analytic in $u$ and $v$. Thus one can shift the integration plane defined by $u=\bar{v}$ to another plane, where (5.25) actually has solutions, but $u=\bar{v}$ is no longer guaranteed. Details can be found in [22]. This means that we have to solve the following set of differential equations:

$$
\dot{u}=-2 s^{2} \frac{\mathrm{i}}{\hbar} \partial_{1} \mathcal{H}(v, u), \quad \dot{v}=2 s^{2} \frac{\mathrm{i}}{\hbar} \partial_{2} \mathcal{H}(v, u)
$$

with boundary conditions

$$
u(0)=z_{I}, \quad v(\tau)=\bar{z}_{F}-2 \pi n .
$$

Note that now the values of $u(\tau)$ and $v(0)$ are not directly given by $v(\tau)$ and $u(0)$, in particular we do not, in general, have $v(0)=\overline{u(0)}$ and $u(\tau)=\overline{v(\tau)}$. Rather, $v(0)$ and $u(\tau)$ 
have to be computed by solving (5.27), they can hence be understood as functions of the initial conditions $u(0)=z_{I}$ and $v(\tau)=\bar{z}_{F}-2 \pi n$.

We now investigate the continuum limit for the different factors in (5.23), starting with the exponential

$$
\begin{aligned}
f^{(\overrightarrow{0}, n)}(\vec{v}, \vec{u})= & 2 \pi n \delta \mathrm{i}+\sum_{k=0}^{N}\left(\frac{u_{k}-v_{k}}{2 s}\right)^{2}-\sum_{k=0}^{N-1}\left[\left(\frac{u_{k}-v_{k+1}}{2 s}\right)^{2}+\frac{\mathrm{i}}{\hbar} \frac{\tau}{N} \mathcal{H}\left(v_{k+1}, u_{k}\right)\right] \\
& -\left(\frac{v_{0}-z_{I}}{2 s}\right)^{2}-\left(\frac{\bar{z}_{F}-2 \pi n-u_{N}}{2 s}\right)^{2} \\
= & 2 \pi n \delta \mathrm{i}+\sum_{k=0}^{N-1}\left[\frac{v_{k+1}-v_{k}}{4 s^{2}} u_{k}-v_{k+1} \frac{u_{k+1}-u_{k}}{4 s^{2}}-\frac{\mathrm{i} \tau}{N \hbar} \mathcal{H}\left(v_{k+1}, u_{k}\right)\right] \\
& -\left(\frac{v_{0}-z_{I}}{2 s}\right)^{2}-\left(\frac{\bar{z}_{F}-2 \pi n-u_{N}}{2 s}\right)^{2}+\frac{v_{0}\left(v_{0}-u_{0}\right)}{4 s^{2}}+\frac{u_{N}\left(u_{N}-v_{N}\right)}{4 s^{2}} \\
(N \rightarrow \infty)=2 & \pi n \delta \mathrm{i}+\int_{0}^{\tau} \mathrm{d} t\left[\frac{\dot{v} u-\dot{u} v}{4 s^{2}}-\frac{\mathrm{i}}{\hbar} \mathcal{H}(v, u)\right]-\left(\frac{v(0)-z_{I}}{2 s}\right)^{2} \\
& -\left(\frac{\bar{z}_{F}-2 \pi n-u(\tau)}{2 s}\right)^{2}+\frac{v(0)\left(v(0)-z_{I}\right)}{4 s^{2}}+\frac{u(\tau)\left(u(\tau)-\bar{z}_{F}+2 \pi n\right)}{4 s^{2}} .
\end{aligned}
$$

We turn to the difference equation (5.24). As $N \rightarrow \infty$, (5.24) becomes a differential equation. We use the expansion $(1+b x)^{-1}(1-a x)^{2}=1-(2 a+b) x+O\left(x^{2}\right)$. As $N$ becomes large, one eventually gets, up to $O\left(N^{-2}\right)$,

$$
\begin{aligned}
X_{k+1}=X_{k}- & X_{k}\left(4 s^{2} \partial_{1} \partial_{2} \mathcal{H}\left(v_{k+1}, u_{k}\right)+8 s^{4} \partial_{2}^{2} H\left(v_{k+1}, u_{k}\right) X_{k}\right) \frac{\tau}{N} \frac{\mathrm{i}}{\hbar} \\
- & \frac{1}{2} \frac{\tau}{N} \frac{\mathrm{i}}{\hbar} \partial_{1}^{2} \mathcal{H}\left(v_{k+1}, u_{k}\right) .
\end{aligned}
$$

As $N \rightarrow \infty$, this becomes

$$
\dot{X}=-4 s^{2} X \frac{\mathrm{i}}{\hbar} \partial_{1} \partial_{2} H-8 s^{4} X^{2} \frac{\mathrm{i}}{\hbar} \partial_{2}^{2} H-\frac{\mathrm{i}}{2 \hbar} \partial_{1}^{2} H,
$$

with boundary condition

$$
X(0)=0 .
$$

Furthermore, we get

$$
\begin{aligned}
\prod_{k=0}^{N-1} \frac{1}{\sqrt{1+8 s^{4} \frac{\tau}{N} \frac{\mathrm{i}}{\hbar} \partial_{2}^{2} \mathcal{H}\left(v_{k+1}, u_{k}\right) X_{k}}} & \exp \left[-\frac{1}{2} \sum_{k=0}^{N-1} \ln \left(1+8 s^{4} \frac{\tau}{N} \frac{\mathrm{i}}{\hbar} \partial_{2}^{2} \mathcal{H}\left(v_{k+1}, u_{k}\right) X_{k}\right)\right] \\
& =\exp \left[\sum_{k=0}^{N-1}\left(-4 \frac{\mathrm{i}}{\hbar} s^{4} \partial_{2}^{2} \mathcal{H}\left(v_{k+1}, u_{k}\right) X_{k}+O\left(N^{-2}\right)\right)\right] \\
(N \rightarrow \infty) & =\exp \left(-4 s^{4} \frac{\mathrm{i}}{\hbar} \int_{0}^{\tau} \mathrm{d} t \partial_{2}^{2} \mathcal{H}(v(t), u(t)) X(t)\right) .
\end{aligned}
$$

To solve the differential equations (5.32), we perturb the boundary data (5.27) around a given solution $v(t), u(t)$ of (5.27) via

$$
\tilde{u}(0)=u(0)+\delta u(0), \quad \tilde{v}(\tau)=v(\tau)+\delta v(\tau),
$$


where one has to determine the difference of this solution with the original, i.e. the evolution of the perturbations $\delta u(t), \delta v(t)$. In first order of the perturbation this yields

$$
\begin{aligned}
& \delta \dot{u}=-2 \mathrm{i} s^{2} \partial_{1}^{2} \mathcal{H}(v, u) \delta v-2 \mathrm{i} s^{2} \partial_{1} \partial_{2} \mathcal{H}(v, u) \delta u \\
& \delta \dot{v}=2 \mathrm{i} s^{2} \partial_{1}^{2} \mathcal{H}(v, u) \delta u+2 \mathrm{i} s^{2} \partial_{1} \partial_{2} \mathcal{H}(v, u) \delta v .
\end{aligned}
$$

Defining now

$$
X(t):=\frac{1}{4 s^{2}} \frac{\delta u(t)}{\delta v(t)},
$$

one then finds with (5.36):

$$
\begin{aligned}
\dot{X} & =\frac{1}{4 s^{2}} \frac{\delta \dot{u}}{\delta v}-\frac{1}{4} \frac{\delta u}{(\delta v)^{2}} \delta \dot{v} \\
& =-\frac{\mathrm{i}}{2 \hbar} \partial_{1}^{2} \mathcal{H}(v, u)-\frac{\mathrm{i}}{\hbar} \frac{\delta u}{\delta v} \partial_{1} \partial_{2} \mathcal{H}(v, u)-\frac{\mathrm{i}}{2 \hbar}\left(\frac{\delta u}{\delta v}\right)^{2} \partial_{2}^{2} \mathcal{H}(v, u) \\
& =-\frac{\mathrm{i}}{2 \hbar} \partial_{1}^{2} \mathcal{H}(v, u)-4 s^{2} \frac{\mathrm{i}}{\hbar} X \partial_{1} \partial_{2} \mathcal{H}(v, u)-8 s^{4} \frac{\mathrm{i}}{\hbar} X^{2} \partial_{2}^{2} \mathcal{H}(v, u) .
\end{aligned}
$$

So this is — for every choice of boundary perturbation (5.35) — a solution of (5.32). The boundary perturbations have to be chosen in a way to satisfy (5.33), which can easily be done by choosing $\delta u(0)=0$, i.e. by only perturbing $v(\tau)$.

With this knowledge, we are now able to rewrite factor (5.34) with the help of (5.36):

$$
2 s^{2} \frac{\delta u}{\delta v} \frac{\mathrm{i}}{\hbar} \partial_{2}^{2} \mathcal{H}(v, u)=\frac{\delta \dot{v}}{\delta v}-2 s^{2} \frac{\mathrm{i}}{\hbar} \partial_{1} \partial_{2} \mathcal{H}(v, u)
$$

and therefore

$$
\begin{gathered}
\exp \left(-4 s^{4} \frac{\mathrm{i}}{\hbar} \int_{0}^{\tau} \mathrm{d} t X \partial_{2}^{2} \mathcal{H}\right)=\exp \left(-\frac{\mathrm{i}}{2 \hbar} \int_{0}^{\tau} \mathrm{d} t \frac{\mathrm{d}}{\mathrm{d} t} \ln \delta \bar{w}+s^{2} \frac{\mathrm{i}}{\hbar} \int_{0}^{\tau} \mathrm{d} t \partial_{1} \partial_{2} \mathcal{H}\right) \\
=\sqrt{\frac{\delta v(0)}{\delta v(\tau)}} \exp \left(s^{2} \frac{\mathrm{i}}{\hbar} \int_{0}^{\tau} \mathrm{d} t \partial_{1} \partial_{2} \mathcal{H}\right) .
\end{gathered}
$$

Combining results (5.29), (5.34) and (5.40), the limit $N \rightarrow \infty$ of (5.23) becomes

$$
\begin{aligned}
\delta\left\langle z_{F}\left|\mathrm{e}^{-\frac{\mathrm{i}}{\hbar} \hat{H} \tau}\right| z_{I}\right\rangle_{\delta} \approx & \sqrt{\frac{\pi}{s^{2}}} \sum_{n \in \mathbb{Z}} \mathrm{e}^{2 \pi \mathrm{i} n \delta}\left\{\sqrt{\frac{\delta v(0)}{\delta v(\tau)}}\right\}_{n} \exp \left\{s^{2} \frac{\mathrm{i}}{\hbar} \int_{0}^{\tau} \mathrm{d} t \partial_{1} \partial_{2} \mathcal{H}\right\}_{n} \\
& \times \exp \left\{\int_{0}^{\tau} \mathrm{d} t\left(\frac{\dot{v} u-\dot{u} v}{4 s^{2}}-\frac{\mathrm{i}}{\hbar} \mathcal{H}(v, u)\right)-\left(\frac{v(0)-z_{I}}{2 s}\right)^{2}\right. \\
& -\left(\frac{\bar{z}_{F}-2 \pi n-u(\tau)}{2 s}\right)^{2}+\frac{v(0)\left(v(0)-z_{I}\right)}{4 s^{2}} \\
& \left.+\frac{u(\tau)\left(u(\tau)-\bar{z}_{F}+2 \pi n\right)}{4 s^{2}}\right\}_{n}
\end{aligned}
$$

Here the subscript $n$ shall remind us of the fact that the propagator is a sum over all $n$, where for each $n$ the complex classical trajectory $u(t), v(t)$ given by (5.27) is different: $u$ starts at $z_{I}$ and $v$ ends at $\bar{z}_{F}-2 \pi n$. The function $X(t)$ for each of these paths has to be computed separately, and at the end all propagators for these paths have to be summed up, each one with a phase $\mathrm{e}^{2 \pi \mathrm{i} n \delta}$. 
As a consistency check we consider the case $\tau \rightarrow 0$. Then the solution of the Hamiltonian equations (5.27) becomes trivial for every $n \in \mathbb{Z}$, in particular $u(t)=z_{I}$ and $v(t)=\bar{z}_{F}-2 \pi n$. With this, (5.41) becomes

$$
\begin{aligned}
\sqrt{\frac{\pi}{s^{2}}} \sum_{n \in \mathbb{Z}} \mathrm{e}^{2 \pi \mathrm{i} n \delta} & \sqrt{1} \exp (0) \exp \left[0-\left(\frac{2 \pi n+\bar{z}_{F}-z_{I}}{2 s}\right)^{2}-\left(\frac{\bar{z}_{F}-2 \pi n-z_{I}}{2 s}\right)^{2}\right. \\
& \left.+\frac{\left(\bar{z}_{F}-2 \pi n\right)\left(\bar{z}_{F}-2 \pi n-z_{I}\right)}{4 s^{2}}+\frac{z_{I}\left(z_{I}-\bar{z}_{F}+2 \pi n\right)}{4 s^{2}}\right] \\
= & \sqrt{\frac{\pi}{s^{2}}} \sum_{n \in \mathbb{Z}} \exp \left[2 \pi \mathrm{i} n \delta-\left(\frac{\bar{z}_{F}-2 \pi n-z_{I}}{2 s}\right)^{2}\right]={ }_{\delta}\left\langle z_{F} \mid z_{I}\right\rangle_{\delta},
\end{aligned}
$$

which is the inner product of two coherent states (3.11).

\subsection{The complex action}

Like in [22], the prefactor with the square root in (5.41) can be rewritten in terms of a complex action. We will do the same here and write, in accordance to [22], the boundary points of the classical trajectories as

$$
\begin{array}{ll}
u^{\prime}:=u(0)=z_{I}, & u^{\prime \prime}:=u(\tau) \\
v^{\prime}:=v(0), & v^{\prime \prime}:=v(\tau)=\bar{z}_{F}-2 \pi n .
\end{array}
$$

Then we define the complex action to be

$S\left(u^{\prime}, v^{\prime \prime}, \tau\right):=\int_{0}^{\tau} \mathrm{d} t\left[\frac{\mathrm{i} \hbar}{4 s^{2}}(\dot{u} v-\dot{v} u)-\mathcal{H}(u, v)\right]-\frac{\mathrm{i} \hbar}{4 s^{2}}\left(u^{\prime} v^{\prime}+u^{\prime \prime} v^{\prime \prime}\right)$,

where the independent variables in $S$ are $u^{\prime}, v^{\prime \prime}$ and $\tau$. The complex trajectories $u(t)$ and $v(t)$ result from the variational principle with $S$ as complex action and are hence functions of $u^{\prime}, v^{\prime \prime}$ and $\tau$. Perturbing these variables, the variation of $S$ is given by

$$
\begin{aligned}
\delta S\left(u^{\prime}, v^{\prime \prime}, \tau\right)= & \int_{0}^{\tau} \mathrm{d} t\left[\left(\frac{\mathrm{i} \hbar}{2 s^{2}} \dot{u}-\partial_{1} \mathcal{H}\right) \delta v-\left(\frac{\mathrm{i} \hbar}{2 s^{2}} \dot{v}-\partial_{2} \mathcal{H}\right) \delta u\right] \\
& -\frac{\mathrm{i} \hbar}{2 s^{2}}\left(v^{\prime} \delta u^{\prime}+u^{\prime \prime} \delta v^{\prime \prime}\right)-\mathcal{H}\left(u^{\prime \prime}, v^{\prime \prime}, \tau\right) \delta \tau .
\end{aligned}
$$

The integral vanishes, since the classical trajectories are defined by the solutions of Hamilton's equations (5.27). Hence we get

$$
\frac{\partial S}{\partial u^{\prime}}=-\frac{\mathrm{i} \hbar}{2 s^{2}} v^{\prime}, \quad \frac{\partial S}{\partial v^{\prime \prime}}=-\frac{\mathrm{i} \hbar}{2 s^{2}} u^{\prime \prime}, \quad \frac{\partial S}{\partial \tau}=-\mathcal{H}\left(u^{\prime \prime}, v^{\prime \prime}, \tau\right) .
$$

The first equation of (5.45) shows that

$$
\frac{\partial^{2} S}{\partial u^{\prime} \partial v^{\prime \prime}}=-\frac{\mathrm{i} \hbar}{2 s^{2}} \frac{\delta v^{\prime}}{\delta v^{\prime \prime}},
$$

and (5.41) can be rewritten in terms of the complex action $S$, which yields the final result for the coherent state propagator (5.1) in the semiclassical approximation:

$$
\begin{aligned}
K_{S^{1}}\left(z_{F}, \tau, z_{I}, 0\right) \approx & \sqrt{2 \pi} \sum_{n \in \mathbb{Z}} \sum_{v} e^{2 \pi \mathrm{i} n \delta} \sqrt{\frac{\hbar}{\mathrm{i}} \frac{\partial^{2} S_{v}}{\partial u^{\prime} \partial v^{\prime \prime}}\left(z_{I}, \bar{z}_{F}-2 \pi n, \tau\right)} \\
& \times \exp \left\{s^{2} \frac{\mathrm{i}}{\hbar} \int_{0}^{\tau} \mathrm{d} t \partial_{1} \partial_{2} \mathcal{H}\right\}_{n, v} \\
& \times \exp \left\{\frac{\mathrm{i}}{\hbar} S_{v}\left(z_{I}, \bar{z}_{F}-2 \pi n, \tau\right)-\frac{z_{I}^{2}+\left(\bar{z}_{F}-2 \pi n\right)^{2}}{4 s^{2}}\right\} .
\end{aligned}
$$


Here the sum over $v$ shall indicate that for each $n$ there may be different complex paths $u$ and $v$ satisfying (5.27) and (5.28). Each of them has to be computed separately and taken into account in formula (5.47).

By comparing result $(5.47)$ for the propagator $K_{S^{1}}$ on the circle with the coherent state propagator $K_{\mathbb{R}}$ for quantum mechanics on the real line [22], one readily sees

$$
K_{S^{1}}\left(z_{F}, \tau, z_{I}, 0\right) \sim \sum_{n \in \mathbb{Z}} \mathrm{e}^{2 \pi n \delta \mathrm{i}} K_{\mathbb{R}}\left(z_{F}-2 \pi n, \tau, z_{I}, 0\right) .
$$

This demonstrates that quantum mechanics on the circle is nothing but a periodic quantum mechanic on the real line, but with a phase shift for each period. The proportionality factor has to be chosen to ensure that both propagators are normalized correctly.

\subsection{Angle representation}

From formula (5.47) on can now obtain, for instance, the angle representation $\left\langle\phi_{F}\left|\mathrm{e}^{-\frac{\mathrm{i}}{\hbar} \hat{H} \tau}\right| \phi_{I}\right\rangle$ by convolution with the coherent states as functions of $\phi$. We just state the final result:

$$
\begin{aligned}
& \left\langle\phi_{F}\left|\mathrm{e}^{-\frac{\mathrm{i}}{\hbar} \hat{H} \tau}\right| \phi_{I}\right\rangle \approx \sqrt{\frac{2 \pi}{s^{2}}} \sum_{n \in \mathbb{Z}} \sum_{\nu} \mathrm{e}^{2 \pi \mathrm{i} n \delta} \frac{1}{\sqrt{\left(m_{q p}\right)_{n, v}}} \\
& \times \exp \left\{s^{2} \frac{\mathrm{i}}{\hbar} \int_{0}^{\tau} \mathrm{d} t \delta \mathcal{H}(\phi, p)\right\}_{n, v} \exp \left\{\frac{\mathrm{i}}{\hbar} \int_{0}^{\tau} \mathrm{d} t \frac{\dot{\phi} p}{s^{2}}-\mathcal{H}(\phi, p)\right\}_{n, v},
\end{aligned}
$$

with

$$
\mathcal{H}(\phi, p)=\frac{{ }_{\delta}\langle\phi+\mathrm{i} p / \hbar|\hat{H}| \phi+\mathrm{i} p / \hbar\rangle_{\delta}}{{ }_{\delta}\langle\phi+\mathrm{i} p / \hbar \mid \phi+\mathrm{i} p / \hbar\rangle_{\delta}} .
$$

The integrals in (5.49) have to be taken over the solutions of the classical trajectories satisfying

$$
\begin{array}{ll}
\dot{\phi}=2 s^{2} \frac{\partial \mathcal{H}}{\partial p}(\phi, p), & \dot{p}=-2 s^{2} \frac{\partial \mathcal{H}}{\partial \phi}(\phi, p) \\
\phi(0)=\phi_{I}, & \phi(\tau)=\phi_{F}-2 \pi n,
\end{array}
$$

and $m_{q p}$ is an entry of the tangent matrix [22] that can be computed from the complex action (5.43). The additional index $v$ indicates that, as already mentioned, even for paths with fixed $n$, there may be more than one classical trajectory satisfying (5.51). If there is, all have to be taken into account. In the angle representation, the sum over $n$ has a nice interpretation. On a circle, a particle can go from $\phi_{I}$ to $\phi_{F}$ in infinitely many ways: the different paths can all differ by their relative winding number. The parameter $\delta$ determines the phase shift the particle acquires by 'going round the circle'. All these paths contribute to the propagator, each one with an additional factor of $\mathrm{e}^{2 \pi \mathrm{in} \delta}$. This demonstrates the fact that the motion of a quantum mechanical particle depends on the global topology of the space it is moving in. The propagator (5.49) takes all these paths into account correctly.

The mathematical realization of this becomes more transparent, when comparing (5.49) to the result for the propagator on the real line [22]:

$$
K_{S^{1}}\left(\phi_{F}, \tau, \phi_{I}, 0\right) \sim \sum_{n \in \mathbb{Z}} \mathrm{e}^{2 \pi \mathrm{i} n \delta} K_{\mathbb{R}}\left(\phi_{F}-2 \pi n, \tau, \phi_{I}, 0\right) .
$$

Like in (5.48), the proportionality factor has to be chosen to normalize both propagators correctly. 


\section{Summary and conclusion}

A brief overview of the complexifier coherent states for quantum mechanics on the circle has been given, summarizing the results from various authors. Furthermore, it was shown that these states are useful for semiclassical analysis, by considering the Bargmann-Segal representation and the semiclassical propagator.

The Bargmann-Segal representation, from which the Husimi distribution can be defined, shows significant differences compared to the one for quantum mechanics on the real line. Periodicity of the system restricts the possible forms of the phase-space wavefunctions $\psi_{\delta}(z)$. In particular, apart from the zeros of $\psi_{\delta}(z)$, the state is completely determined by the choice of normalization and an additional integer. This is in contrast to the situation on the real line, where, apart from the zeros of the phase-space wavefunction $\psi(z)$, one has to specify normalization and two arbitrary complex coefficients to reconstruct the state. Furthermore, on the real line, the coherent states are the only states whose Husimi distribution has no zeros at all. On the circle, the eigenvectors $|n\rangle_{\delta}$ of the momentum operator $\hat{p}$ are the only vectors whose Husimi distribution is without zeros. This demonstrates the qualitatively different behaviour of the two kinds of coherent states, which results from the different topologies of phase space.

The semiclassical propagator for the complexifier coherent states was derived. The result is the infinite sum over coherent state propagators on the real line, each one evaluated for a path with a different winding number. Also, each of the single propagators acquires a phase which is determined by the parameter $\delta$ of the representation. This shows the influence of the global phase-space topology on the motion of a quantum mechanical particle. In contrast to the real line, a wavefunction moving on a circle can interfere with itself because of the non-simply connected configuration space. The result also illustrates the physical meaning of the representation parameter $\delta$ : it determines the phase shift the wavefunction acquires when moving around the circle. The parameter $\delta$ can be chosen according to the problem at hand and has to be taken into account when computing the coherent state propagator.

These results show that the complexifier coherent states for $G=U(1)$ are in principle as useful for a semiclassical analysis of periodic systems as the harmonic oscillator coherent states are for systems on the real line.

\section{Acknowledgments}

BB would like to thank Thomas Thiemann for vivid discussions about the semiclassical propagator. The authors would like to thank Hans Kastrup for pointing out the significance of reference [2].

\section{Appendix A. Hadamard decomposition of the sine}

In this section, we will prove formula (4.15). We start by noting that the sine function is of first order:

$$
\left|\sin \frac{z-a}{2}\right| \leqslant \exp \left(\frac{1}{2}|a|+\frac{1}{2}|z|\right) .
$$

So, the function $z \mapsto \sin \frac{z-a}{2}$ can, according to the Hadamard decomposition, be written [33] in the following way: 


$$
\begin{aligned}
\sin \frac{z-a}{2} & =\mathrm{e}^{C_{0}+C_{1} z} \prod_{n \in \mathbb{Z}}\left(1-\frac{z}{a+2 \pi n}\right) \exp \left(\frac{z}{a+2 \pi n}\right) \\
& =\mathrm{e}^{C_{0}+C_{1} z}\left(1-\frac{z}{a}\right) \mathrm{e}^{\frac{z}{a}} \prod_{n=1}^{\infty}\left(1-\frac{z}{a+2 \pi n}\right)\left(1-\frac{z}{a-2 \pi n}\right) \exp \left(\frac{z}{a+2 \pi n}+\frac{z}{a-2 \pi n}\right) \\
& =\mathrm{e}^{C_{0}+\widetilde{C}_{1} z}\left(1-\frac{z}{a}\right) \prod_{n=1}^{\infty}\left(1-\frac{z}{a+2 \pi n}\right)\left(1-\frac{z}{a-2 \pi n}\right)
\end{aligned}
$$

with

$$
\widetilde{C_{1}}:=C_{1}+\frac{1}{a}+\sum_{n=1}^{\infty} \frac{2 a}{a^{2}-4 \pi^{2} n^{2}} .
$$

By setting $z=0$, one gets the condition $\mathrm{e}^{C_{0}}=-\sin \frac{a}{2}$, and from the anti-periodicity of the sine,

$$
\begin{aligned}
-1=\frac{\sin \frac{z+2 \pi-a}{2}}{\sin \frac{z-a}{2}} & =\mathrm{e}^{2 \pi \widetilde{C_{1}}} \prod_{n \in \mathbb{Z}} \frac{1-\frac{z+2 \pi}{a+2 \pi n}}{1-\frac{z}{a+2 \pi n}} \\
& =\mathrm{e}^{2 \pi \widetilde{C_{1}}} \lim _{N \rightarrow \infty} \prod_{n=-N}^{N} \frac{a+2 \pi(n-1)-z}{a+2 \pi n-z} \\
& =\mathrm{e}^{2 \pi \widetilde{C_{1}}} \lim _{N \rightarrow \infty} \frac{a-2 \pi(N+1)-z}{a+2 \pi N-z} \\
& =-\mathrm{e}^{2 \pi \widetilde{C_{1}}} .
\end{aligned}
$$

Thus, $\mathrm{e}^{2 \pi \widetilde{C_{1}}}=1$ and $\widetilde{C_{1}}=\mathrm{i} k$, with $k$ being an integer. This means that

$\sin \frac{z-a}{2}=-\sin \frac{a}{2} \mathrm{e}^{\mathrm{i} k z}\left(1-\frac{z}{a}\right) \prod_{n=1}^{\infty}\left(1-\frac{z}{a+2 \pi n}\right)\left(1-\frac{z}{a-2 \pi n}\right)$.

We will now show that $k$ is actually zero. To achieve this, we set $z=2 a$, and obtain

$$
\sin \frac{a}{2}=\sin \frac{a}{2} \mathrm{e}^{2 \mathrm{i} k a} \prod_{n=1}^{\infty}\left(1-\frac{2 a}{a+2 \pi n}\right)\left(1-\frac{2 a}{a-2 \pi n}\right),
$$

and therefore

$$
\begin{aligned}
\mathrm{e}^{-2 \mathrm{i} k a} & =\prod_{n=1}^{\infty}\left(1-\frac{2 a}{a+2 \pi n}\right)\left(1-\frac{2 a}{a-2 \pi n}\right) \\
& =\prod_{n=1}^{\infty} \frac{2 \pi n-a}{2 \pi n+a} \frac{-2 \pi n-a}{a-2 \pi n}=1,
\end{aligned}
$$

which immediately shows that, if $k \neq 0, a=\pi m / k$ for some $m \in Z$. But then the function $z \mapsto \sin \frac{z-a}{2}$ is real for $z \in \mathbb{R}$, which, as can be seen by (A.5), is only possible for $k=0$. So we have

$$
\sin \frac{z-a}{2}=-\sin \frac{a}{2}\left(1-\frac{z}{a}\right) \prod_{n=1}^{\infty}\left(1-\frac{z}{a+2 \pi n}\right)\left(1-\frac{z}{a-2 \pi n}\right)
$$

which is formula (4.15). 


\section{Appendix B. Proof of a technical lemma}

In this section, the proof of lemma 4.1 will be delivered.

Let $\left\{a_{k}\right\}$ be a sequence in $([0,2 \pi) \times \mathrm{i} \mathbb{R}) \backslash\{0\}$ of order 1 . Let $v_{k}:=\operatorname{sgn} \operatorname{Im} a_{k}$ be the sign of the imaginary part of $a_{k}$ (with sgn $0:=1$ ). Then

$$
\prod_{k}\left[-\exp \left(\pi \cot \frac{a_{k}}{2}\right)\right]=\exp \left[\sum_{k} \pi\left(\cot \frac{a_{k}}{2}+v_{k} \mathrm{i}\right)\right] .
$$

Proof. We would like to employ the formula

$$
\prod_{k} f_{k}=\exp \left[\sum_{k} \ln f_{k}\right] .
$$

To use this formula for complex numbers $f_{k}$, one has to select a branch cut of the logarithm. The particular choice of the cut results in different imaginary parts of (some of the) $\ln f_{k}$, differing by an integer multiple of $2 \pi \mathrm{i}$. The exponential of these different $\ln f_{k}$ hence is the same for all choices of branch cut, which means that (B.2) is valid for every choice of branch cut of the logarithm. In the following, we choose the cut on the negative real axis, i.e.

$$
\ln \exp (\mathrm{i} x)=\left\{\begin{array}{ll} 
& \vdots \\
\mathrm{i} x+2 \pi \mathrm{i} & x \in(-3 \pi,-\pi] \\
\mathrm{i} x & x \in(-\pi, \pi] \\
\mathrm{i} x-2 \pi \mathrm{i} & x \in(\pi, 3 \pi] \\
& \vdots
\end{array} .\right.
$$

So, we have to compute $\ln \left(-\exp \left(\pi \cot a_{k} / 2\right)\right)$. The result crucially depends on the imaginary part of $\alpha_{k}$, so we need to keep track of it.

Since the sequence $\left\{a_{k}\right\}_{k}$ is of order 1 , it has no accumulation points. So, since $a_{k} \in$ $[0,2 \pi) \times \mathrm{i} \mathbb{R}$, there is only a finite number of $a_{k}$ with a fixed real part. Thus we can order the sequence $\left\{a_{k}\right\}_{k}$ by ascending imaginary part, i.e.

$$
\operatorname{Im} a_{k} \leqslant \operatorname{Im} a_{l} \Leftrightarrow k<l .
$$

The number of $a_{k}$ can be finite or infinite, which leaves three possibilities. The index $k$ ranges from $-\infty$ to 0 , from 0 to $\infty$, or from $-\infty$ to $\infty$, depending on the distribution of the $a_{k}$ over the strip. We will concentrate on the last of these four cases, which is the most general one. The argument for the other three cases runs along similar lines.

So, the $a_{k}$ go to i $\infty$ for $k \rightarrow \infty$ and to $-\mathrm{i} \infty$ for $k \rightarrow \infty$. Since $\operatorname{Re} a_{k} \in[0,2 \pi)$, we have

$$
\lim _{k \rightarrow \pm \infty} \operatorname{Im} \cot \frac{a_{k}}{2}=\mp 1,
$$

which follows from the properties of the cotangent. So there are $N, M \in \mathbb{Z}$ such that

$$
\begin{aligned}
& \operatorname{Im} \cot \frac{a_{k}}{2} \in\left(\frac{1}{2}, \frac{3}{2}\right), \quad \operatorname{Im} a_{k}<0 \quad \text { for all } \quad k<N, \\
& \operatorname{Im} \cot \frac{a_{k}}{2} \in\left(-\frac{3}{2},-\frac{1}{2}\right), \quad \operatorname{Im} a_{k}>0 \quad \text { for all } \quad k>M .
\end{aligned}
$$


Hence, with the definition of the logarithm (B.3), we have

$$
\ln \left[-\exp \left(\pi \cot \frac{a_{k}}{2}\right)\right]= \begin{cases}\pi \cot \frac{a_{k}}{2}-\mathrm{i} \pi, & k<N \\ \pi \cot \frac{a_{k}}{2}+\mathrm{i} \pi, & k>M .\end{cases}
$$

This means that, with $v_{k}:=\operatorname{sgn} \operatorname{Im} a_{k}$,

$$
\ln \left[-\exp \left(\pi \cot \frac{a_{k}}{2}\right)\right]=\pi\left(\cot \frac{a_{k}}{2}+\mathrm{i} v_{k}\right)
$$

for all $k$, up to finitely many exceptions. So, there is an integer $K \in \mathbb{Z}$, such that

$$
\sum_{k \in \mathbb{Z}} \ln \left[-\exp \left(\pi \cot \frac{a_{k}}{2}\right)\right]=\sum_{k \in \mathbb{Z}} \pi\left(\cot \frac{a_{k}}{2}+\mathrm{i} v_{k}\right)+2 \pi \mathrm{i} K .
$$

From this it follows immediately that

$$
\begin{array}{r}
\prod_{k \in \mathbb{Z}}\left[-\exp \left(\pi \cot \frac{a_{k}}{2}\right)\right]=\exp \sum_{k \in \mathbb{Z}} \ln \left[-\exp \left(\pi \cot \frac{a_{k}}{2}\right)\right] \\
=\exp \left[\sum_{k} \pi\left(\cot \frac{a_{k}}{2}+v_{k} \mathrm{i}\right)+2 \pi \mathrm{i} K\right]=\exp \sum_{k} \pi\left(\cot \frac{a_{k}}{2}+v_{k} \mathrm{i}\right),
\end{array}
$$

as claimed in the lemma.

Note that the lemma stays true, of course, if one replaces $v_{k}=\operatorname{sgn} \operatorname{Im} a_{k}$ by any other sequence $\tilde{v}_{k} \in\{ \pm 1, \pm 3, \pm 5, \ldots\}$, that differs from $v_{k}$ at not more than finitely many $k$ s. Only the sum is shifted by an integer multiple of $2 \pi \mathrm{i}$, which does not show up in the exponential.

But in the case of infinitely many $a_{k} \mathrm{~s}$, the choice of the $v_{k}=\operatorname{sgn} \operatorname{Im} a_{k}$ (up to finitely many exceptions) is important for the sum to converge, that is, one is not allowed to change the $v_{k}$ at more that finitely many $k \mathrm{~s}$, in order for the sum $\sum_{k} \pi\left(\cot \frac{a_{k}}{2}+v_{k} \mathrm{i}\right)$ to exist at all. The proof that the sum then actually converges rests on the fact that the sequence $\left\{a_{k}\right\}$ is of order 1 .

The lemma is only true if we define the sign of 0 to be either 1 or -1 . For real $a_{k}$, for which the imaginary part of $\cot a_{k} / 2$ vanishes, one has

$$
\ln \left[-\exp \left(\pi \cot \frac{a_{k}}{2}\right)\right]=\pi\left(\cot \frac{a_{k}}{2} \pm \mathrm{i}\right),
$$

the sign depending on the particular choice of the branch cut. So, for real $a_{k}$, one has to have either $v_{k}=+1$ of $v_{k}=-1$, although $\operatorname{sgn} \operatorname{Im} a_{k}=0$. Thus, we adjust the definition of the sign of 0 to be +1 , and with this definition the lemma is thoroughly true. Since there are only finitely many real $a_{k}$, we could have chosen the sign of 0 to be -1 as well, for the reasons stated above.

\section{References}

[1] Korsch H J and Mossmann S 2003 Phys. Lett. A 31754

[2] Kastrup H 2006 Phys. Rev. A 73052104

[3] Kowalski K, Rembieliński J and Papaloucas L C 1996 J. Phys. A: Math. Gen. 294149

[4] Kowalski K and Rembieliński J 2002 J. Phys. A: Math. Gen. 351405

[5] Kowalski K and Rembieliński J 2004 J. Phys. A: Math. Gen. 3711447

[6] Zhang W, Feng D H and Gilmore R 1990 Rev. Mod. Phys. 62867

[7] Thiemann T 2002 Complexifier coherent states for quantum general relativity Preprint gr-qc/0206037

[8] Hall B 1994 J. Funct. Anal. 122103

[9] Hall B 1997 J. Funct. Anal. 14398 
[10] Hall B 1997 Commun. Math. Phys. 184233

[11] Thiemann T 2001 Class. Quantum Grav. 182025

[12] Thiemann T and Winkler O 2001 Class. Quantum Grav. 182561

[13] Thiemann T and Winkler O 2001 Class. Quantum Grav. 184629

[14] Ashtekar A, Bojowald M and Lewandowski J 2003 Adv. Theor. Math. Phys. 7233

[15] Chang S-J and Shi K-J 1986 Phys. Rev. A 347

[16] Gonzáles J A and del Olmo M A 1998 J. Phys. A: Math. Gen. 318841

[17] Nonnenmacher S and Voros A 1998 J. Stat. Phys. 92431

[18] Hall B and Mitchell J 2002 J. Math. Phys. 431211

[19] Gorin T, Korsch H J and Mirbach B 1997 Chem. Phys. 217145

[20] Korsch H J, Müller C and Wiescher H 1997 J. Phys. A: Math. Gen. 30 L677

[21] Ruzzi M, Marchiolli M A, da Silva E C and Galetti D 2006 J. Phys. A: Math. Gen. 399881

[22] Baranger M, de Aguiar M A M, Keck F, Korsch H J and Schellhaaß B 2001 J. Phys. A: Math. Gen. 347227 see also Baranger M, de Aguiar M A M, Keck F, Korsch H J and Schellhaaß B 2002 J. Phys. A: Math. Gen. 359493

Baranger M, de Aguiar M A M, Keck F, Korsch H J and Schellhaaß B 2003 J. Phys. A: Math. Gen. 369795

[23] Schulman L 1968 Phys. Rev. 1761558

[24] Kowalski K and Rembieliński J 2002 Phys. Lett. A 293109

[25] Parisio F and de Aguiar M A M 2003 Phys. Rev. A 68062112

[26] Ribeiro A D, de Aguiar M A M and Baranger M 2004 Phys. Rev. E 69066204

[27] Novaes M and de Aguiar M A M 2005 Phys. Rev. A 72032105

[28] Ribeiro A D, Novaes M and de Aguiar M A M 2005 Phys. Rev. Lett. 95050405

[29] Ribeiro A D, de Aguiar M A M and de Toledo Piza A F R 2006 J. Phys. A: Math. Gen. 393085

[30] Friedlander G and Joshi M 1998 Introduction to the Theory of Distributions 2nd edn (Cambridge: Cambridge University Press)

[31] Vourdas A 2006 J. Phys. A: Math. Gen. 39 R65

[32] Wiescher H and Korsch H J 1997 J. Phys. A: Math. Gen. 301763

[33] Rubel L and Colliander J 1995 Entire and Meromorphic Functions (Berlin: Springer)

[34] Apostol T M 1990 Modular Functions and Dirichlet Series in Number Theory (New York: Springer) 